17

\title{
Влияние дифракции на импульс сжатого света в протоколе многомодовой резонансной квантовой памяти на тепловом атомном ансамбле
}

\author{
(С) Э.Р. Зинатуллин, К.С. Тихонов , Т.Ю. Голубева, Ю.М. Голубев
}

Санкт-Петербургский государственный университет, 199034 Санкт-Петербург, Россия

"e-mail: tikhonov.kyril@gmail.com

Поступило в редакцию 20.05.2020 г.

В окончательной редакции 22.05.2020 г.

Принято к публикации 05.06.2020 г.

Рассмотрено влияние дифракции на сохранение квантового состояния импульса квадратурно-сжатого света в ансамбле теплых атомов для коллинеарной конфигурации световых полей. Движение атомов приводит к тому, что даже в случае прямого считывания, когда и сигнальное, и управляющее поля распространяются в том же направлении, что и при записи, возникает нескомпенсированный фазовый набег. Этот фазовый набег приводит к тому, что к сжатой квадратуре импульса света подмешивается растянутая. В результате степень сжатия импульса может существенно уменьшиться. В работе проведен анализ влияния дифракции для разных конфигураций протокола многомодовой резонансной квантовой памяти и найдены способы, как свести это влияние к возможному минимуму.

Ключевые слова: сжатый свет, квантовая память, тепловое движение атомов.

DOI: $10.21883 /$ OS.2020.09.49876.159-20

\section{1. Введение}

При анализе работы протоколов многомодовой квантовой памяти для непрерывных переменных очень часто используется приближение неподвижных атомов [1-5]. С практической точки зрения такое приближение является вполне оправданным, если в качестве среды использовать, например, примеси в кристаллах [6] или глубоко охлажденные атомы, движением которых за время хранения можно пренебречь [7]. Однако каждую из этих физических систем довольно трудно масштабировать, что ограничивает область их возможного применения в квантово-информационных приложениях [1]. В связи с этим в качестве альтернативы были предложены протоколы квантовой памяти, в которых в качестве атомной среды выступают теплые пары щелочных металлов [8-12]. Для таких протоколов приближение неподвижных атомов оказывается уже неприменимым.

Использование ансамбля теплых атомов в протоколах квантовой памяти существенно отличается от использования холодных и сопряжено с рядом особенностей. Сюда можно отнести эффект Допплера, а также ряд других нежелательных процессов, связанных с выходом атомов ансамбля из области взаимодействия со световыми полями, их соударением друг с другом и со стенками оптической кюветы. Все эти явления достаточно хорошо изучены, и предложен ряд способов, как можно свести их влияние к минимуму [13].

В нашей работе мы остановимся на другой важной особенности протоколов квантовой памяти на тепловом атомном ансамбле. В теоретических работах $[14,15]$ бы- ло показано, что из-за движения атомов распределение спиновой когерентности, которая образуется в ячейке памяти при записи, будет постоянно меняться даже тогда, когда все поля выключены. Такое „размытие“, спиновой когерентности приводит к изменению модового состава ячейки памяти, что в свою очередь сказывается как на сохранении квантово-статистических свойств записанного сигнала, так и на процедуре оптимизации протокола. Это было подтверждено экспериментально в работе [16].

Мы рассмотрим сохранение квадратурно-сжатого импульса света внутри ячейки быстрой резонансной многомодовой квантовой памяти [17]. Однако теперь в отличие от работы [15] нас будет интересовать не только продольное движение атомов, но и поперечное. При этом особое внимание уделим учету дифракции при распространении импульсов света в атомной среде. Отметим, что в большинстве теоретических работ, посвященных квантовой памяти, обычно рассматривают случай обратного считывания, так как эффективность в этом случае оказывается выше [18], чем для прямого. При этом влиянием дифракции, как правило, пренебрегают, считая, что оптическая толщина среды небольшая и возникающий набег фазы несущественен. Однако в недавних экспериментальных работах [19] была получена оптическая толщина, которая на порядок превосходит используемые ранее теоретические оценки (порядка 1500). Большая оптическая толщина способна обеспечить высокую эффективность протокола памяти [20], но при этом фазовый набег при распространении света сквозь среду будет также большим и 
пренебречь дифракцией уже будет нельзя. Кроме того, наличие даже небольшого фазового набега, вызванного дифракцией, может сказаться на записи и восстановлении квадратурно-сжатого света, так как в этом случае к сжатой квадратуре будет подмешиваться растянутая, что приведет либо к ухудшению квантово-статистических свойств сигнального поля, либо к их полной потере.

Мы построим теоретическую модель быстрой резонансной многомодовой квантовой памяти, в которой на этапах записи и считывания сжатого светового импульса на атомную среду учтем влияние дифракции. Тепловое движение будем рассматривать только на этапе хранения, считая, что этапы записи и считывания короткие и за это время атомы смещаются незначительно. При этом по-отдельности рассмотрим случай продольного и случай поперечного движений атомов среды, предполагая, что движение по другим координатам было подавлено с помощью техники лазерного охлаждения [21]. Такой подход позволит разделить различные процессы, влияющие на квантовое хранение света, и указать наиболее существенные из них. Для этого мы проведем анализ решений уравнений движения Гейзенберга и затем проверим все наши выводы с помощью численного расчета. В разд. 5, основываясь на полученных результатах, мы предложим способы оптимизации протокола памяти.

\section{2. Дифракция в квантовой памяти на тепловом ансамбле атомов}

\section{1. Модель резонансной быстрой квантовой памяти}

В этом разделе мы приведем краткое описание протокола быстрой резонансной квантовой памяти, который основан на одновременном взаимодействии импульсов квантового сигнального и классического управляющего световых полей с ансамблем атомов, имеющих $\Lambda$-конфигурацию энергетических уровней.

Мы решаем трехмерную пространственную задачу, и для удобства вычислений будем считать, что все атомы ансамбля расположены в бесконечном плоском слое толщины $L$, перпендикулярном к оси $z$, вдоль которой распространяются импульсы сигнального и управляющего световых полей на этапе записи (рис. 1, $a$ ). На этапе считывания импульс управляющего поля может быть направлен как вдоль оси $z$ (рис. $1, c$ ), так и в противоположном ей направлении (рис. $1, b$ ). Рассмотрим каждый из этих случаев, и согласно устоявшейся терминологии будем ссылаться на них, как на „прямое“ и „обратное“ считывание соответственно.

Считаем, что в начальный момент времени $(t=0)$ все атомы с помощью оптической накачки приготовлены в состоянии $|1\rangle$ (рис. 2,a). Затем в процессе записи сигнальное поле переводит часть атомов ансамбля в возбужденное состояние $|3\rangle$, а сильное управляющее поле переносит эти атомы из состояния $|3\rangle$ в состояние

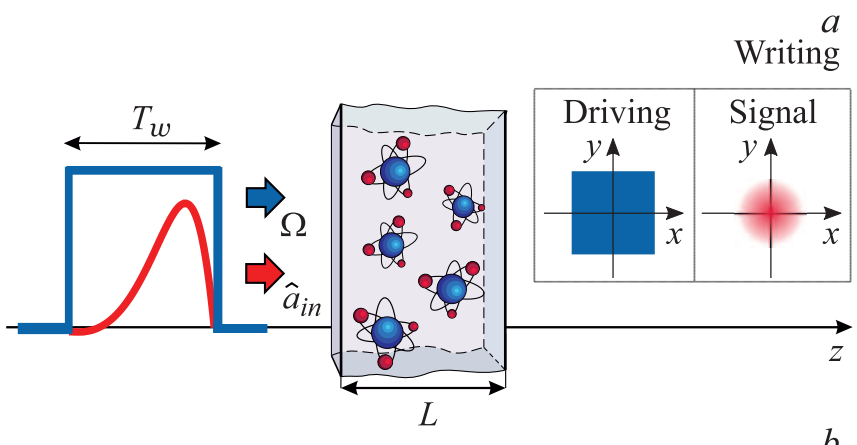

$b$
ard

Backward

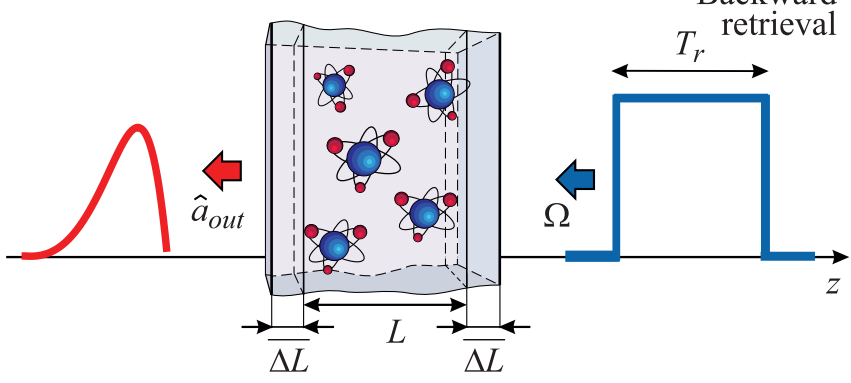

Forward

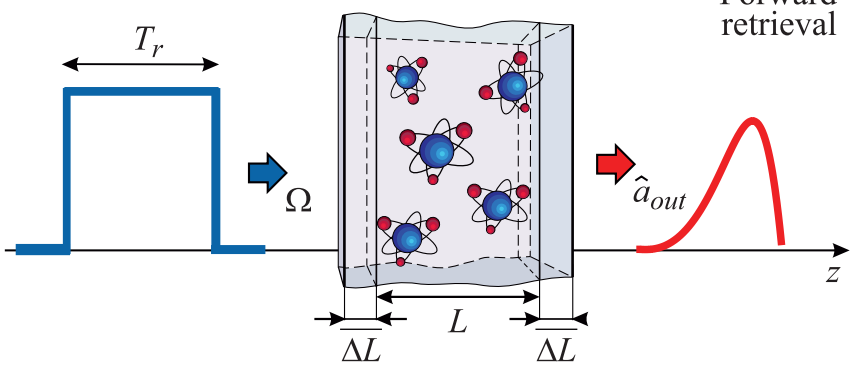

Рис. 1. Геометрическая конфигурация действующих в протоколе световых полей: $(a)$ при записи; $(b)$ при обратном считывании; (c) при прямом считывании. На вставке представлен их поперечный профиль: управляющее поле - плоская волна; сигнальное поле - гауссов пучок.

$|2\rangle$. В результате между уровнями $|1\rangle$ и $|2\rangle$ образуется спиновая когерентность (спиновая волна), которая затем хранится в ячейке памяти вплоть до момента считывания. Именно эта когерентность является долгоживущей степенью свободы атомного ансамбля, на которую отпечатываются квантово- статистические свойства сигнального поля. Наконец, на этапе считывания протокол завершается тем, что на вход ячейки памяти (либо вдоль оси $z$, либо в противоположном ей направлении) подается импульс управляющего поля, который переносит атомы обратно с уровня $|2\rangle$ на уровень $|3\rangle$. Это приводит к индуцированному переходу с уровня $|3\rangle$ на уровень $|1\rangle$ с восстановлением квантового сигнального импульса, поданного на вход ячейки. Отметим, что все указанные выше поля действуют только на своих переходах и находятся с ними в резонансе, т.е. $\omega_{s}=\omega_{13}$ и $\omega_{d}=\omega_{23}$, где $\omega_{s}$ и $\omega_{d}-$ несущие частоты сигнального и управляющего световых полей, а $\omega_{13}$ и 


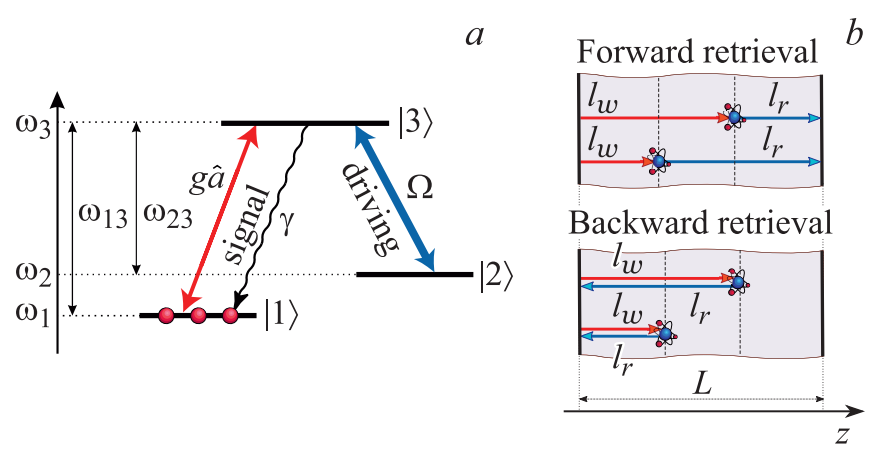

Рис. 2. (a) Энергетическая схема уровней атома ансамбля с сигнальным и управляющим полями, действующими на оптическом переходе $|1\rangle-|3\rangle$ и $|2\rangle-|3\rangle$ соответственно; (b) схематическое объяснение дифракции в ансамбле неподвижных атомов. $l_{w}$ и $l_{r}-$ длины оптических путей при записи и при считывании. При обратном считывании полный оптический путь $\left(l_{w}+l_{r}\right)$ для каждой точки среды зависит от координаты $z$.

$\omega_{23}$ - частоты соответствующих переходов. Уровни $|1\rangle$ и $|2\rangle$ выбираются метастабильными.

В нашей работе мы будем учитывать влияние дифракции, возникающей при распространении световых импульсов внутри атомной среды. Обратимся к рис. 2, $b$, и для простоты рассмотрим сначала неподвижные атомы. Длина оптического пути $l$ при распространении световых импульсов вдоль оси $z$ в процессе памяти складывается из двух участков $l_{w}$ и $l_{r}$, относящихся к этапам записи и считывания, т.е. $l=l_{w}+l_{r}$. Конец участка $l_{w}$ (и начало участка $l_{r}$ ) физически соответствует месту, где происходит взаимодействие атомов среды с фотонами сигнального и управляющего световых импульсов. При этом, так как взаимодействие с определенной вероятностью может произойти в любом месте внутри ячейки, для случая обратного считывания полная длина оптического пути $l$ будет зависеть от точки $z$ (места взаимодействия), тогда как для прямого считывания она всегда будет постоянной, т.е. $l=L$. Таким образом, для двух любых точек атомной среды, расположенных на разных расстояниях вдоль оси $z$, при обратном считывании возникнет разность длин оптических путей, которая приведет к деструктивному проявлению дифракции при восстановлении сигнального поля. Для прямого считывания влияние дифракции сведется лишь к набегу постоянной фазы, который может быть легко компенсирован с помощью обычной фазовой пластинки. Однако, если вместо холодного атомного ансамбля взять теплый, движение атомов среды приведет к тому, что даже в случае прямого считывания длину оптических путей уже нельзя будет считать постоянной. Как мы увидим в дальнейшем, это также приведет к деструктивному проявлению дифракции.

Кроме дифракции, в нашей модели памяти мы также учтем тепловое движение атомов среды. Для этого нам будет удобно рассмотреть по-отдельности случай продольного (относительно оси $z$ ) движения атомов и случай их поперечного движения (перпендикулярно оси $z$ ). При этом будем предполагать, что в каждом из этих случаев движение по сопряженным координатам было подавлено с помощью техники лазерного охлаждения [21]. Это существенно упростит расчеты и, главное, позволит выявить, влияние какого движения продольного или поперечного - является наиболее деструктивным по отношению к записанному в ячейку памяти импульсу света. Подчеркнем, что в нашей модели мы будем учитывать движение только на этапе хранения длительностью $T_{s}$, считая, что длительности этапов записи $T_{w}$ и считывания $T_{r}$ по сравнению с ним достаточно короткие и атомы не успевают существенно сместиться. Кроме того, будем считать, что выполняется следующее условие:

$$
T_{w}, T_{r} \ll \gamma^{-1} \ll T_{s},
$$

где $\gamma^{-1}$ - время спонтанного распада с уровня $|3\rangle$ на уровень $|1\rangle$.

Процесс взаимодействия сигнального и управляющего световых импульсов с атомами среды будем описывать с помощью гамильтониана взаимодействия, записанного в коллективных переменных с использованием дипольного приближения и приближения вращающейся волны [17]:

$$
\hat{V}(t)=i \hbar \int_{V} d^{3} \mathbf{r}\left[\left(g \hat{a}(\mathbf{r}, t) \hat{\sigma}_{31}(\mathbf{r}, t)+\Omega \hat{\sigma}_{32}(\mathbf{r}, t)\right)-h . c .\right] .
$$

Интегрирование ведется по радиусу-вектору r, указывающему место, в котором происходит взаимодействие световых импульсов и атомов ансамбля, при этом $\mathbf{r}=(\boldsymbol{\rho}, z)$, где $z-$ продольная координата, а $\boldsymbol{\rho}-$ поперечная составляющая $\mathbf{r}$. Сила взаимодействия ансамбля атомов с квантовым сигнальным полем определяется константой связи $g$, а с классическим полем, которое в нашем случае является плоской монохроматической волной - частотой Раби $\Omega$ :

$$
g=\sqrt{\frac{\omega_{s}}{2 \varepsilon_{0} \hbar c}} d_{31}, \quad \Omega=\sqrt{\frac{\omega_{d}}{2 \varepsilon_{0} \hbar c}} d_{32}
$$

где $d_{31}$ и $d_{32}$ - матричные элементы оператора дипольного момента, отвечающие соответствующим переходам. Для простоты будем считать и константу связи, и частоту Раби вещественными.

Оператор уничтожения $\hat{a}(\mathbf{r}, t)$ соответствует медленной амплитуде квантового сигнального поля и подчиняется следующим коммутационным соотношениям [22], записанным с использованием приближений параксиальности и квазимонохроматичности:

$$
\begin{aligned}
& {\left[\hat{a}(\boldsymbol{\rho}, z, t), \hat{a}^{\dagger}\left(\boldsymbol{\rho}^{\prime}, z^{\prime}, t\right)\right]} \\
& =c\left(1-\frac{i}{k_{s}} \frac{\partial}{\partial z}-\frac{1}{2 k_{s}^{2}} \Delta \boldsymbol{\rho}\right) \delta^{3}\left(\mathbf{r}-\mathbf{r}^{\prime}\right)
\end{aligned}
$$




$$
\left[\hat{a}(\boldsymbol{\rho}, z, t), \hat{a}^{\dagger}\left(\boldsymbol{\rho}, z, t^{\prime}\right)\right]=\delta\left(t-t^{\prime}\right),
$$

где $k_{s}$ - волновое число для импульса сигнального поля.

Оператор $\hat{\sigma}_{m n}(\mathbf{r}, t)$ описывает коллективную когерентность, возникающую в результате дипольных переходов между состояниями $|m\rangle$ и $|n\rangle$. Для таких операторов выполняются коммутационные соотношения

$$
\left[\hat{\sigma}_{m n}(\mathbf{r}, t), \hat{\sigma}_{n m}\left(\mathbf{r}^{\prime}, t\right)\right]=\left(\hat{N}_{m}(\mathbf{r}, t)-\hat{N}_{n}(\mathbf{r}, t)\right) \delta^{3}\left(\mathbf{r}-\mathbf{r}^{\prime}\right),
$$

которые связывают их с коллективными операторами заселенностей $\hat{N}_{m}$ и $\hat{N}_{n}$ состояний $|m\rangle$ и $|n\rangle$.

\section{2. Уравнения движения}

Из гамильтониана (2) для этапов записи и считывания, используя коммутационные соотношения (4), (5) и (6), можно получить полную систему уравнений движения Гейзенберга, что было сделано в работах [18]. Нас, впрочем, будет интересовать не вся система уравнений, а лишь ее часть, которая образует замкнутую систему линейных дифференциальных уравнений.

Напомним, что сигнальное квантовое поле, действующее на переходе между состояниями $|1\rangle$ и $|3\rangle$, является слабым, т.е. содержит мало фотонов, а значит, среднее число атомов $\left\langle\hat{N}_{1}\right\rangle$ в состоянии $|1\rangle$, в котором изначально приготовлен весь ансамбль, со временем практически не меняется. Классическое поле действует на другом переходе и никак не влияет на заселенность состояния $|1\rangle$. Это позволяет считать, что выполняется следующее неравенство:

$$
\left\langle\hat{N}_{1}\right\rangle \gg\left\langle\hat{N}_{2}\right\rangle,\left\langle\hat{N}_{3}\right\rangle,
$$

и полностью пренебречь заселенностями состояний $|2\rangle$ и $|3\rangle$, а оператор заселенности $\hat{N}_{1}$ заменить вещественным числом $N$, которое соответствует объемной концентрации атомов ансамбля в области взаимодействия со световыми полями.

Как следствие, полную систему уравнений можно линеаризовать и затем совершить преобразование Холштейна-Примакова, заменив операторы когерентности $\hat{\sigma}_{12}$ и $\hat{\sigma}_{13}$ новыми операторами атомной среды $\hat{b}$ и $\hat{c}$ :

$$
\begin{aligned}
& \hat{b}(\boldsymbol{\rho}, z, t)=\frac{1}{\sqrt{N}} \hat{\sigma}_{12}(\boldsymbol{\rho}, z, t), \\
& \hat{c}(\boldsymbol{\rho}, z, t)=\frac{1}{\sqrt{N}} \hat{\sigma}_{13}(\boldsymbol{\rho}, z, t),
\end{aligned}
$$

которые подчиняются бозонным коммутационным соотношениям:

$$
\begin{aligned}
& {\left[\hat{b}(\boldsymbol{\rho}, z, t), \hat{b}^{\dagger}\left(\boldsymbol{\rho}^{\prime}, z^{\prime}, t\right)\right]=\left[\hat{c}(\boldsymbol{\rho}, z, t), \hat{c}^{\dagger}\left(\boldsymbol{\rho}^{\prime}, z^{\prime}, t\right)\right]} \\
& =\delta^{2}\left(\boldsymbol{\rho}-\boldsymbol{\rho}^{\prime}\right) \delta\left(z-z^{\prime}\right)
\end{aligned}
$$

Таким образом, так же, как и оператор поля $\hat{a}$, эти операторы описывают квантовые гармонические осцилляторы, но относящиеся уже не к световому полю, а к атомной среде.

Далее нам удобно совершить переход к безразмерным переменным:

$$
\Omega t \rightarrow t, \quad \frac{2 g^{2} N}{\Omega} z \rightarrow z, \quad \frac{2 g^{2} N}{\Omega}|\boldsymbol{\rho}| \rightarrow|\boldsymbol{\rho}|,
$$

в которых время выражено в единицах частоты Раби, а продольная и поперечные координаты в единицах эффективной оптической толщины, которая отличается от естественной в $\Omega / \gamma$ раз.

В итоге получили замкнутую систему дифференциальных уравнений Гейзенберга для медленных безразмерных амплитуд, описывающих эволюцию рассматриваемой системы на этапах записи и считывания:

$$
\begin{gathered}
\left(\frac{\partial}{\partial z}+\frac{i}{2 k_{s}} \Delta \boldsymbol{\rho}\right) \hat{a}(\boldsymbol{\rho}, z, t)=-\frac{1}{\sqrt{2}} \hat{c}(\boldsymbol{\rho}, z, t), \\
\frac{\partial}{\partial t} \hat{b}(\boldsymbol{\rho}, z, t)=-\hat{c}(\boldsymbol{\rho}, z, t), \\
\frac{\partial}{\partial t} \hat{c}(\boldsymbol{\rho}, z, t)=\frac{1}{\sqrt{2}} \hat{a}(\boldsymbol{\rho}, z, t)+\hat{b}(\boldsymbol{\rho}, z, t) .
\end{gathered}
$$

В левой части уравнения для поля $\hat{a}(\boldsymbol{\rho}, z, t)$ мы пренебрегли производной по времени, которая описывает распространение волнового фронта, предполагая, что длительность импульсов $T_{w}$ и $T_{r}$ намного больше времени прохождения света через ячейку памяти $L / c$, т. е. $T_{w}, T_{r} \gg L / c$. При этом учли вторые производные по поперечным координатам, которые, как мы увидим дальше, связаны с дифракцией света при его распространении через атомную среду.

Подчеркнем, что в системе уравнений (11) и везде далее все переменные и операторы являются безразмерными величинами. Безразмерные операторы могут быть получены из коммутационных соотношений (4), (5), (9) с использованием формул для перехода к безразмерным величинам (10).

На этапе хранения, как и в работе [15], будет удобно перейти от рассмотрения полного ансамбля атомов с объемной концетрацией атомов $N$ к подансамблю с объемной концентрацией атомов $N_{\boldsymbol{v}}$, который движется как целое из начального положения полного ансамбля в некотором заданном направлении с заданной скоростью v. Как уже было сказано ранее, мы по-отдельности рассмотрим случаи продольного и поперечного движения атомов. Для продольного движения уравнение, описывающее эволюцию спиновой когерентности подансамбля на этапе хранения, будет иметь следующий вид:

$$
\left(\frac{\partial}{\partial t}+v_{z} \frac{\partial}{\partial z}\right) \hat{b}\left(\boldsymbol{\rho}, z, t ; v_{z}\right)=0,
$$

где $v_{z}-$ скорость продольного движения подансамбля. Аналогичное уравнение имеет место и для поперечного 
движения:

$$
\left(\frac{\partial}{\partial t}+\mathbf{v}_{\rho} \nabla_{\rho}\right) \hat{b}\left(\boldsymbol{\rho}, z, t ; \mathbf{v}_{\rho}\right)=0
$$

где $\mathbf{v}_{\rho}-$ скорость поперечного движения подансамбля.

Здесь стоит сказать, что на этапе хранения именно $\hat{b}(\boldsymbol{\rho}, z, t)$ содержит квантово-статистическую информацию сигнального импульса $\hat{a}(\boldsymbol{\rho}, z, t)$, тогда как $\hat{c}(\boldsymbol{\rho}, z, t)$ из-за спонтанного распада состояния $|3\rangle$ этой информации не содержит.

\section{3. Общее решение}

Решение системы уравнений Гейзенберга для этапов записи и считывания (11) можно провести с помощью прямого и обратного преобразований Лапласа [18]. Для этого сначала удобно совершить преобразование Фурье и перейти от поперечной координаты $\boldsymbol{\rho}$ к связанной с ней координате q в обратном пространстве. В результате спиновая когерентность $\hat{b}\left(\mathbf{q}, z, T_{w}\right)$, которая образуется к концу этапа записи, будет связана с полем $\hat{a}_{i n}(\mathbf{q}, t)$ на входе в ячейку памяти (при $z=0$ ) следующим выражением:

$$
\begin{aligned}
\hat{b}\left(\mathbf{q}, z, T_{w}\right)= & -e^{-i \frac{\mathbf{q}^{2}}{2 k_{s}} z} \int_{0}^{T_{w}} d t G_{a b}(z, t) \hat{a}_{i n}\left(\mathbf{q}, T_{w}-t\right) \\
& +\operatorname{vac}\left(T_{w}\right)
\end{aligned}
$$

где $\operatorname{vac}\left(T_{w}\right)$ - это вакуумный вклад на этапе записи, a $G_{a b}(z, t)$ - ядро интегрального преобразования для этапа записи.

При прямом считывании спиновая когерентность $\hat{b}\left(\mathbf{q}, z, T_{w}+T_{s}\right)$, которая остается в системе к концу этапа хранения, превращается в восстановленное поле $\hat{a}_{\text {out }}^{\text {for }}(\mathbf{q}, t)$ на выходе из ячейки (при $\left.z=L\right)$ :

$$
\begin{aligned}
\hat{a}_{\text {out }}^{\text {for }}(\mathbf{q}, t)= & -\int_{0}^{L} d z e^{-i \frac{\mathrm{q}^{2}}{2 k_{s}}(L-z)} G_{b a}(L-z, t) \\
& \times \hat{b}\left(\mathbf{q}, z, T_{w}+T_{s}\right)+v a c^{\text {for }}\left(T_{r}\right),
\end{aligned}
$$

где $v a c^{\text {for }}\left(T_{r}\right)$ - вакуумный вклад при прямом считывании. Ядро интегрального преобразования $G_{b a}(z, t)$ совпадает с ядром интегрального преобразования $G_{a b}(z, t)$ в $(14)$, т. е. $G_{a b}(z, t)=G_{b a}(z, t)$. При обратном считывании спиновая когерентность $\hat{b}\left(\mathbf{q}, z, T_{w}+T_{s}\right)$ превращается в восстановленное поле $\hat{a}_{\text {out }}^{\text {back }}(\mathbf{q}, t)$ на выходе из ячейки (при $z=0)$ :

$$
\begin{aligned}
\hat{a}_{\mathrm{out}}^{\mathrm{back}}(\mathbf{q}, t)= & -\int_{0}^{L} d z e^{-i \frac{\mathrm{q}^{2}}{2 k_{s}} z} G_{b a}(z, t) \\
& \times \hat{b}\left(\mathbf{q}, z, T_{w}+T_{s}\right)+v a c^{\mathrm{back}}\left(T_{r}\right),
\end{aligned}
$$

где $v a c^{\text {back }}\left(T_{r}\right)$ - вакуумный вклад при обратном считывании.
Отметим, что в полученных выражениях экспоненциальные множители возникают из-за второй производной по пространственной координате в уравнении для поля в системе (11), и именно они отвечают за эффект дифракции.

Из уравнений (12) и (13) следует, что спиновая когерентность $\hat{b}$ подансамбля к началу этапа считывания (при $t=T_{w}+T_{s}$ ) в случае продольного движения всех его атомов с общей скоростью $v_{z}$ будет связана со спиновой когерентностью в конце этапа записи (при $\left.t=T_{w}\right)$ :

$$
\hat{b}\left(\boldsymbol{\rho}, z, T_{w}+T_{s} ; v_{z}\right)=\hat{b}\left(\boldsymbol{\rho}, z-v_{z} T_{s}, T_{w} ; v_{z}\right) .
$$

Аналогичное решение получим и для случая поперечного движения атомов подансамбля со скоростью $\mathbf{v}_{\rho}$ :

$$
\hat{b}\left(\boldsymbol{\rho}, z, T_{w}+T_{s} ; \mathbf{v}_{\rho}\right)=\hat{b}\left(\boldsymbol{\rho}-\mathbf{v}_{\rho} T_{s}, z, T_{w} ; \mathbf{v}_{\rho}\right) .
$$

Далее, чтобы перейти от решений для подансамблей атомов к решению для полного ансамбля, будем считать, что температура атомов $\tau$ превышает критическую температуру $\tau_{c}$ :

$$
\tau \gg \tau_{c}=\frac{n^{2 / 3} h^{2}}{3 m k_{b}},
$$

где $n$ - объемная концентрация, $m$ - масса одной частицы, $k_{b}$ - постоянная Больцмана, $h-$ постоянная Планка. Тогда можно полагать, что к началу этапа записи в ансамбле установилось термодинамическое равновесие, которое мы можем описать с помощью максвелловского распределения по скоростям.

В результате после усреднения решений (17) и (18) с максвелловским распределением по скоростям мы получим выражение для спиновой когерентности всего ансамбля в случае продольного движения атомов:

$$
\begin{aligned}
\hat{b}\left(\boldsymbol{\rho}, z, T_{w}+T_{s} ; \overline{\Delta L}\right)= & \frac{T_{s}}{\sqrt{\pi} \overline{\Delta L}} \int_{\mathbf{R}} d v_{z} \exp \left(-\frac{v_{z}^{2} T_{s}^{2}}{\overline{\Delta L}^{2}}\right) \\
& \times \hat{b}\left(\boldsymbol{\rho}, z-v_{z} T_{s}, T_{w}\right) .
\end{aligned}
$$

Здесь $\overline{\Delta L}=T_{s} u_{z}-$ среднее смещение атомов в продольном направлении на этапе хранения со средней скоростью $u_{z}$. Аналогичное выражение получим и для спиновой когерентности в случае поперечного движения атомов:

$$
\begin{aligned}
\hat{b}\left(\boldsymbol{\rho}, z T_{w}+T_{s} ; \overline{\Delta w}\right)= & \frac{T_{s}^{2}}{\pi \overline{\Delta w}^{2}} \int_{\mathbf{R}^{2}} d \mathbf{v}_{\rho} \exp \left(-\frac{\mathbf{v}_{\rho}^{2} T_{s}^{2}}{\overline{\Delta w}^{2}}\right) \\
& \times \hat{b}\left(\boldsymbol{\rho}-\mathbf{v}_{\rho} T_{s}, z, T_{w}\right),
\end{aligned}
$$

где $\overline{\Delta w}=u_{\rho} T_{s}-$ среднее смещение атомов при поперечном тепловом движении; $u_{\rho}-$ средняя скорость атомов в поперечной плоскости в произвольном выбранном направлении. 
Мы записали решения (20) и (21) для когерентности на этапе хранения с помощью поперечной координаты $\rho$, а решения (14), (15) и (16) для когерентности и поля на этапах записи и считывания - с помощью поперечной координаты в обратном пространстве q. Напомним, что переход от прямого пространства к обратному осуществляется через обычное преобразование Фурье. В дальнейшем будет удобно пользоваться решениями, записанными как для прямого пространства, так и для обратного, и мы будем отмечать это, указывая на соответствующую координату. При этом будем иметь в виду, что были выполнены все необходимые преобразования Фурье.

\section{4. Моды Шмидта}

Дальнейший анализ полученных решений удобно проводить на языке мод Шмидта [23].

Будем сначала считать, что атомы ансамбля неподвижны на протяжении полного цикла памяти. В этом случае спиновая когерентность $\hat{b}$, которая образуется внутри среды к концу этапа записи, на протяжении этапа хранения остается неизменной. Тогда поле на входе в ячейку памяти оказывается связанным с полем на выходе из нее через интегральное уравнение:

$$
\hat{a}_{\text {out }}(\mathbf{q}, t)=\int_{0}^{T_{w}} d t^{\prime} G\left(t, t^{\prime}\right) \hat{a}_{\mathrm{in}}\left(\mathbf{q}, T_{w}-t^{\prime}\right)+v a c,
$$

где $G\left(t, t^{\prime}\right)$ - ядро этого преобразования. Для прямого считывания оно имеет вид

$$
G^{\mathrm{for}}\left(t, t^{\prime}, \mathbf{q}\right)=e^{-i \frac{\mathrm{q}^{2}}{k_{s}} L} \int_{0}^{L} d z G_{b a}(z, t) G_{a b}\left(L-z, t^{\prime}\right),
$$

а для обратного

$$
G^{\text {back }}\left(t, t^{\prime}, \mathbf{q}\right)=\int_{0}^{L} d z e^{-i \frac{q^{2}}{k_{s}} z} G_{b a}(z, t) G_{a b}\left(z, t^{\prime}\right) .
$$

Далее возьмем длительности импульсов при записи и при считывании равными друг другу, т.е. $T_{w}=T_{r}$ и положим $\mathbf{q}=0$. В этом случае получившиеся ядра оказываются симметричными относительно перестановки аргументов $t$ и $t^{\prime}$, и их можно переписать в виде разложения Шмидта:

$$
G\left(t, t^{\prime}, \mathbf{q}=0\right)=\sum_{i=1}^{\infty} \sqrt{\lambda_{i}} \psi_{i}(t) \psi_{i}\left(t^{\prime}\right),
$$

где $\left\{\psi_{i}(t)\right\}-$ полный ортонормированный набор собственных функций этого ядра, a $\left\{\sqrt{\lambda_{i}}\right\}-$ соответствующие этим функциям собственные значения.

Примечательным свойством найденных собственных функций $\left\{\psi_{i}(t)\right\}$ является то, что в случае, когда атомы неподвижны и дифракцией можно пренебречь $\left(\mathbf{q}^{2} L / k_{s} \ll 1\right)$, сигнальное поле с временным профилем, совпадающим с любой из этих собственных функций (например, $\left.\psi_{j}(t)\right)$, будет сохранено внутри ячейки памяти и затем восстановлено без каких-либо искажений профиля. Эффективность протокола при этом будет равна квадрату собственного числа (т.е. $\lambda_{j}$ ), которое соответствует данной собственной функции. Это делает их весьма удобным инструментом при сравнительном анализе влияния как теплового движения, так и дифракции на рассматриваемый протокол памяти.

Отметим теперь другую особенность разложения Шмидта. Поскольку $\left\{\psi_{i}(t)\right\}-$ полный ортонормированный набор функций, определенных при $t \in\left[0, T_{w}\right]$, то любой сигнальный импульс $\hat{a}(t)$ длительностью $T_{w}$ может быть представлен в виде разложения по этому набору:

$$
\hat{a}(t)=\sum_{i=1}^{\infty} \psi_{i}(t) \hat{e}_{i},
$$

где $j$-й коэффициент $\hat{e}_{j}-$ это бозонный оператор уничтожения фотона для $j$-го квантового гармонического осциллятора (моды) с временным профилем $\psi_{j}(t)$, а $n_{j}=\left\langle\hat{e}_{j}^{\dagger} \hat{e}_{j}\right\rangle-$ среднее число фотонов в $j$-й моде. Таким образом, если в разложении ядра по модам Шмидта больше одного члена с ненулевыми собственными числами, то сигнальное поле внутри ячейки памяти будет вести себя как суперпозиция этих мод. И если хотя бы два члена этой суперпозиции (например, $l$-й и $m$-й) будут невакуумными (т.е. $n_{l}, n_{m}>0$ ), то относительно ячейки памяти (т.е. в базисе собственных мод $\left.\left\{\psi_{i}(t)\right\}\right)$ такое поле будет многомодовым, даже если оно было получено от одномодового источника.

Кроме собственных функций полного цикла памяти, для дальнейшего анализа нам также понадобятся моды среды $\left\{g_{i}(z)\right\}$, которые представляют собой пространственные профили спиновых когерентностей (спиновых волн), образующихся в ячейке памяти, когда на ее вход подано поле в виде одной из собственных функций:

$$
\sqrt[4]{\lambda_{i}} g_{i}(z)=\int_{0}^{T_{w}} d t G_{a b}(z, t) \psi_{i}(t) .
$$

Моды среды также образуют полный ортонормированный набор, что позволяет представить ядро интегрального преобразования (14) в виде ряда

$$
G_{a b}(z, t)=\sum_{i=1}^{\infty} \sqrt[4]{\lambda_{i}} g_{i}(z) \psi_{i}(t)
$$

Отсюда следует, что каждой $j$-ой полевой моде $\psi_{j}(t)$ будет отвечать своя мода среды $g_{j}(z)$, т.е. в процессе записи каждый полевой квантовый осциллятор будет преобразован в атомный (спиновую волну). Отметим, что в силу ортогональности мод среды $\left\{g_{i}(z)\right\}$ при отсутствии теплового движения и дифракции эти осцилляторы будут развиваться независимо друг от друга, т.е. 
их пространственные профили (распределение спиновой когерентности) будут оставаться неизменными.

Это также позволяет нам связать разложение в ряд (26) сигнального поля $\hat{a}(t)$ по собственным функциям $\left\{\psi_{i}(t)\right\}$ с разложением спиновой когерентности $\hat{b}(z)$, которая образуется в ячейке памяти при записи поля $\hat{a}(t)$, по модам среды $\left\{g_{i}(z)\right\}$ :

$$
\hat{b}(z)=\sum_{i=1}^{\infty} g_{i}(z) \hat{e}_{i},
$$

т. е. имеет место взаимно-однозначное соответствие между полевыми модами $\left\{\psi_{i}(t)\right\}$ и модами среды $\left\{g_{i}(z)\right\}$.

Таким образом, в выражении (20) усреднение по продольным скоростям можно проводить для функций отклика $\left\{g_{i}(z)\right\}$, чем мы и воспользуемся в дальнейшем.

\section{5. Перемешивание мод Шмидта при тепловом движении атомов и дифракции}

Перейдем теперь к ситуации, когда мы учитываем и дифракцию, и тепловое движение атомов среды на этапе хранения.

Рассмотрим сначала случай продольного движения атомов. Размытие спиновой когерентности приводит к изменению фазовых зависимостей между модами. В частности, при прямом считывании больше нельзя считать, что дифракция сводится к набегу постоянной фазы как в (23) для неподвижных атомов. Экспоненциальные множители, зависящие от продольной координаты $z$ в выражениях (14) и (15), более не компенсируют друг друга. Из-за дифракции при записи когерентности возникает набег фазы. Используя (20), запишем выражение для моды среды $g_{i}(z)$ к концу этапа хранения при наличии продольного движения атомов среды и с учетом дифракции:

$$
\begin{aligned}
g_{i}(\mathbf{q}, z ; \overline{\Delta L})= & \frac{T_{s}}{\sqrt{\pi} \overline{\Delta L}} \int_{-\infty}^{+\infty} d v_{z} \exp \left(-\frac{v_{z}^{2} T_{s}^{2}}{\overline{\Delta L}^{2}}\right) \\
& \times e^{-i \frac{\mathrm{q}^{2}}{2 k_{s}} z} g_{i}\left(z-v_{z} T_{s}\right) .
\end{aligned}
$$

Запишем теперь ядро для полуцикла памяти, включающего в себя и этап записи, и этап хранения:

$$
G_{a b}(\mathbf{q}, z, t ; \overline{\Delta L})=\sum_{i=1}^{\infty} \sqrt[4]{\lambda_{i}} g_{i}(\mathbf{q}, z ; \overline{\Delta L}) \psi_{i}(t),
$$

а также ядро $G\left(\mathbf{q}, t, t^{\prime} ; \overline{\Delta L}\right)$ для полного цикла памяти, в котором мы учли продольное тепловое движение атомов среды и дифракцию. Для случая прямого считывания оно имеет вид

$$
\begin{aligned}
G^{\mathrm{for}}\left(\mathbf{q}, t, t^{\prime} ; \overline{\Delta L}\right)= & \left.\int_{0}^{L} d z e^{-i \frac{\mathbf{q}^{2}}{2 k_{s}} z} G_{b a}(z, t)\right) \\
& \times G_{a b}\left(\mathbf{q}, L-z, t^{\prime} ; \overline{\Delta L}\right),
\end{aligned}
$$

а для обратного считывания

$$
\begin{aligned}
G^{\text {back }}\left(\mathbf{q}, t, t^{\prime} ; \overline{\Delta L}\right)= & \int_{0}^{L} d z e^{-i \frac{\mathrm{q}^{2}}{2 k_{s}} z} G_{b a}(z, t) \\
& \times G_{a b}\left(\mathbf{q}, z, t^{\prime} ; \overline{\Delta L}\right) .
\end{aligned}
$$

Используя теперь (28) и (31), можем переписать каждое из полученных выражений для ядра полного цикла в виде ряда

$$
G\left(\mathbf{q}, t, t^{\prime} ; \overline{\Delta L}\right)=\sum_{i, j=1}^{\infty} \sqrt[4]{\lambda_{i} \lambda_{j}} F_{i j}(\mathbf{q}, \overline{\Delta L}) \psi_{i}(t) \psi_{j}\left(t^{\prime}\right)
$$

где функция $F_{i j}(\mathbf{q}, \overline{\Delta L})$ описывает перекрывание (или перемешивание) мод среды вследствие дифракции и продольного теплового движения. Для случая прямого считывания она равна

$$
F_{i j}^{\text {for }}(\mathbf{q}, \overline{\Delta L})=\int_{0}^{L} d z e^{-i \frac{\mathbf{q}^{2}}{2 k_{s}} z} g_{i}(z) g_{j}(\mathbf{q}, L-z ; \overline{\Delta L}),
$$

а для обратного считывания

$$
F_{i j}^{\mathrm{back}}(\mathbf{q}, \overline{\Delta L})=\int_{0}^{L} d z e^{-i \frac{\mathrm{q}^{2}}{2 k_{s}} z} g_{i}(z) g_{j}(\mathbf{q}, z ; \overline{\Delta L}) .
$$

Как видим, продольное тепловое движение и дифракция будут перемешивать моды среды как при прямом считывании, так и при обратном. Кроме того, комплексная часть полученных выражений приведет к тому, что в интегральном преобразовании для полного цикла памяти (22) квадратура $\hat{x}_{\text {out }}$ (аналогично и $\hat{y}_{\text {out }}$ ) поля на выходе будет связана с квадратурами $\hat{x}_{\text {in }}$ и $\hat{y}_{\text {in }}$ поля на входе в ячейку (квадратуры введены как $\hat{a}=\hat{x}+i \hat{y}$ ). Таким образом, наличие дифракции сводится не только к перемешиванию мод среды, но и к перемешиванию квадратур сигнального поля, что необходимо учитывать при дальнейшем детектировании восстановленного сигнала.

Аналогичные выражения нетрудно получить и тогда, когда мы рассматриваем поперечное движение атомов на этапе хранения. Однако вместо общего случая мы рассмотрим важный частный случай, когда сигнальное поле, поданное на вход ячейки памяти является одномодовым в поперечной плоскости и представляет собой гауссов пучок с профилем

$$
f(\boldsymbol{\rho})=\sqrt{\frac{2}{\pi w_{0}^{2}}} e^{-\frac{\boldsymbol{\rho}^{2}}{w_{0}^{2}}}
$$

и полушириной $w_{0}$. Для такого поля зависимость от поперечной координаты $\boldsymbol{\rho}$ будет содержаться только в функции $f(\boldsymbol{\rho})$, т. е. $\hat{a}(\boldsymbol{\rho}, t) \rightarrow f(\boldsymbol{\rho}) \hat{a}(t)$. Это приводит к 
тому, что после усреднения по поперечному движению в (21) мы получим

$$
\hat{b}\left(\mathbf{q}, z, T_{w}+T_{s} ; \overline{\Delta w}\right)=\sqrt{\frac{S}{S^{\prime}}} \exp \left(-\frac{\mathbf{q}^{2} \overline{\Delta w}^{2}}{4}\right) \hat{b}\left(\mathbf{q}, z, T_{w}\right) .
$$

Здесь нормировочный множитель $\sqrt{S / S^{\prime}}-$ корень из отношения площади $S$ поперечного профиля сигнального поля на входе в ячейку к площади $S^{\prime}$ на выходе из нее. Наличие этого множителя объясняется сохранением потока (при отсутствии потерь) сигнального поля при его записи и считывании. Зависимость от q когерентности $\hat{b}\left(\mathbf{q}, z, T_{w}\right)$ в правой части выражения будет связана с наличием дифракции.

Заметим, что, поскольку на входе в ячейку памяти сигнальное поле имело гауссов профиль (37), на выходе из ячейки поперечный профиль восстановленного сигнального поля также будет гауссовой функцией, но с большей полушириной $w_{0}^{2}+\overline{\Delta w}^{2}$ :

$$
f(\boldsymbol{\rho} ; \overline{\Delta w})=\sqrt{\frac{2}{\pi\left(w_{0}^{2}+\overline{\Delta w}^{2}\right)}} e^{-\frac{\boldsymbol{\rho}^{2}}{w_{0}^{2}+\overline{\Delta w}^{2}}} .
$$

Таким образом, если потерь нет, то поток фотонов должен остаться прежним, но уже взятый по большей площади. Именно этим и вызвано наличие нормировочного множителя $\sqrt{S / S^{\prime}}$.

В итоге получим выражение для ядра полного цикла памяти при наличии дифракции и поперечного теплового движения:

$$
\begin{aligned}
G\left(\mathbf{q}, t, t^{\prime} ; \overline{\Delta w}\right)= & \sqrt{\frac{S}{S^{\prime}}} f(\mathbf{q} ; \overline{\Delta w}) \\
& \times \sum_{i, j=1}^{\infty} \sqrt[4]{\lambda_{i} \lambda_{j}} F_{i j}(\mathbf{q}) \psi_{i}(t) \psi_{j}\left(t^{\prime}\right),
\end{aligned}
$$

где функция $F_{i j}(\mathbf{q})$ описывает перекрывание мод среды вследствие дифракции:

$$
\begin{gathered}
F_{i j}^{\text {for }}(\mathbf{q})=e^{-i \frac{q^{2}}{2 k_{s}} L} \int_{0}^{L} d z g_{i}(z) g_{j}(L-z), \\
F_{i j}^{\text {back }}(\mathbf{q})=\int_{0}^{L} d z e^{-i \frac{q^{2}}{k_{s}} z} g_{i}(z) g_{j}(z) .
\end{gathered}
$$

Таким образом, мы записали все интересующие нас выражения для ядер полного цикла при наличии дифракции как для продольного, так и для поперечного движения атомов среды на этапе хранения. Из этих общих выражений нетрудно получить выражения для частных случаев, когда мы учитываем только дифракцию или только тепловое движение. Это понадобится для проведения сравнительного анализа и определения роли каждого из этих факторов в рассматриваемом нами протоколе квантовой памяти.

\section{3. Квадратурно-сжатое излучение и его детектирование}

\section{1. Источники квадратурно-сжатого света}

В нашей работе в качестве источников квадратурносжатого излучения рассмотрим простейший одномодовый модельный источник сжатого света и одномодовый субпуассоновский лазер с захватом фазы [24]. Отметим, что первый источник соответствует чистому квантовому состоянию (сжатому), а второй - смешанному. Кроме того, они будут отличаться временными профилями.

\subsection{1. Модельный одномодовый источник сжа-} того света. Будем считать, что временной профиль поля $\hat{a}_{\text {in }}(\boldsymbol{\rho}, t)$ одномодового модельного источника на входе в ячейку памяти совпадает с одной из собственных функций $\left\{\psi_{i}(t)\right\}$ :

$$
\hat{a}_{\text {in }}(\boldsymbol{\rho}, t)=f(\boldsymbol{\rho}) \psi_{k}(t) \hat{e}_{k}+v a c .
$$

Здесь $k$ - номер записываемой моды, а $\hat{e}_{k}$ - оператор уничтожения фотонов для этой моды, $f(\boldsymbol{\rho})$ - гауссов профиль (37) в поперечной плоскости, vac - вакуумный вклад от остальных световых мод. Будем считать, что квадратурная компонента $\hat{x}_{k}$ данного квантового гармонического осциллятора сжата, а компонента $\hat{y}_{k}$, наоборот, растянута $\left(\hat{x}_{k}=\left(\hat{e}_{k}+\hat{e}_{k}^{\dagger}\right) / 2 \hat{y}_{k}=\left(\hat{e}_{k}-\hat{e}_{k}^{\dagger}\right) / 2 i\right)$ :

$$
\left\langle\hat{x}_{k}^{2}\right\rangle=\frac{1}{4} e^{-r}, \quad\left\langle\hat{y}_{k}^{2}\right\rangle=\frac{1}{4} e^{r}
$$

где $r$ - степень сжатия (растяжения). Выбор такого источника обусловлен сравнительной простотой его анализа, сочетающейся с хорошей наглядностью происходящих в ячейке памяти процессов.

Как уже было сказано ранее, сигнальное поле с временным профилем, совпадающим с одной из собственных функций $\left\{\psi_{i}(t)\right\}$, при отсутствии дифракции и теплового движения распространяется внутри ячейки памяти без искажений, и поэтому с ним удобно проводить сравнения результатов, полученных для конфигурации памяти, учитывающей влияние этих негативных факторов. Кроме того, даже для такого одномодового источника будет проявляться перемешивание мод и квадратур из-за наличия дифракции и теплового движения атомов, о которых мы говорили выше. Наличие ненулевых интегралов перекрывания $F_{i j}$ вследствие дифракции и теплового движения приводит к тому, что излучение из одной моды среды будет перераспределяться по другим ее модам, а наличие комплексных компонент к перемешиванию сжатой и растянутой квадратур.

Также стоит отметить, что в работе [15], посвященной анализу зависимости эффективности ячейки квантовой памяти от продольного теплового движения атомов, было показано, что излучение, временной профиль которого совпадает с $\psi_{l}(t)$ одной из собственных функций ячейки $\left\{\psi_{i}(t)\right\}$, при наличии движения сохраняется тем 
лучше, чем проще пространственная структура отвечающей этому профилю моды среды $g_{l}(z)$, т.е. чем более гладкой является эта функция и чем меньше узлов она содержит. Такие моды среды будут в меньшей степени подвержены размытию продольным тепловым движением атомов, чем моды со сложной структурой. Однако в цитируемой работе было рассмотрено только продольное движение атомов среды на этапе хранения для случая обратного считывания. Мы продолжим этот анализ для других конфигураций памяти и посмотрим, какие моды лучше сохраняют квадратурное сжатие записанного излучения.

\subsection{2. Одномодовый субпуассоновский лазер} c захватом фазы. В качестве второго источника выберем субпуассоновский лазер с захватом фазы, работающий в импульсном режиме [24], у которого $\hat{x}$-квадратура является сжатой, а $\hat{y}$-квадратура - растянутой:

$$
\begin{gathered}
\left\langle: \delta \hat{x}(t) \delta \hat{x}\left(t^{\prime}\right):\right\rangle=-\frac{p}{8} \frac{1-\mu}{1-\mu / 2} e^{-\kappa(1-\mu / 2)\left|t-t^{\prime}\right|} \theta(t), \\
\left\langle: \delta \hat{y}(t) \delta \hat{y}\left(t^{\prime}\right):\right\rangle=\frac{1-\mu}{\mu} e^{-\kappa \mu / 2\left|t-t^{\prime}\right|} \theta(t) .
\end{gathered}
$$

Эти выражения записаны с учетом нормального порядка операторов; в них $p$ - параметр накачки, $\mu=\sqrt{n_{0} / n}-$ доля среднего числа фотонов синхронизующего поля $n_{0}$ от общего числа фотонов $n$ в излучении, а $\theta(t)-$ оконная функция, равная единице при $t \in\left[0, T_{w}\right]$ и равная нулю при остальных значениях $t$. Подчеркнем, что это одномодовый источник с временным профилем в виде оконной функции, однако в базисе собственных функций $\left\{\psi_{i}(t)\right\}$, если среди них нет такой же оконной функции, он уже будет являться многомодовым. Как и в случае одномодового источника, рассмотренного ранее, будем считать, что излучение от субпуассоновского лазера в поперечной плоскости будет представлять собой гауссов пучок с профилем (37).

Из выражения (45) мы можем получить корреляционные функции для квадратур $j$-ой и $l$-ой моды:

$$
\begin{aligned}
\left\langle: \delta \hat{x}_{j} \delta \hat{x}_{l}:\right\rangle= & \int_{0}^{T_{w}} \int_{0}^{T_{w}} d t d t^{\prime}\left\langle: \delta \hat{x}\left(T_{w}-t\right) \delta \hat{x}\left(T_{w}-t^{\prime}\right):\right\rangle \\
& \times \psi_{j}(t) \psi_{l}\left(t^{\prime}\right) .
\end{aligned}
$$

Эти функции окажутся удобным инструментом при обсуждении сохранения степени сжатия света.

\section{2. Гомодинное детектирование сигнала от модельного источника}

Перед тем, как мы перейдем к анализу разных режимов работы рассматриваемого протокола квантовой памяти, остановимся на процедуре балансного гомодинного детектирования квадратурно-сжатого излучения, которое получим на выходе из ячейки памяти.
При гомодинировании сигнального поля $\hat{a}_{\text {out }}(\boldsymbol{\rho}, t) \mathrm{c}$ локальным осциллятором $L(\rho, t)$ фототок будет задаваться следующим выражением:

$$
\hat{i}(t)=\iint_{-\infty}^{+\infty} d \boldsymbol{\rho} \hat{a}_{\text {out }}(\boldsymbol{\rho}, t) L^{*}(\boldsymbol{\rho}, t)+\text { h.c. }
$$

В случае модельного одномодового источника, описанного выше, нас будет интересовать степень сжатия этой моды как целого. Для этого будем следить за фототоком на нулевой частоте

$$
\hat{i}_{0}=\frac{1}{\sqrt{T_{w}}} \int_{0}^{T_{w}} d t \hat{i}(t),
$$

а наблюдаемой величиной при этом будет $\left\langle\hat{i}_{0}^{2}\right\rangle$. Рассмотрим несколько важных для нас режимов работы протокола и покажем на их примере, как осуществляется подбор правильной формы локального осциллятора.

Отметим, что почти все выражения, которые мы получим, будут справедливы как для прямого, так и для обратного считывания, поэтому здесь и далее без необходимости не будем оговаривать отдельно, о каком направлении считывания идет речь.

\subsection{1. Без учета дифракции и теплового дви-} жения. В отсутствие каких-либо негативных эффектов при прохождении ячейки памяти амплитуда поля с профилем одной из собственных функций $\left\{\psi_{i}(t)\right\}$ будет уменьшаться пропорционально отвечающему этой функции собственному числу, но сам временной профиль при этом останется неизменным. Другими словами, изменения будут носить масштабный характер. Для $k$-й собственной функции мы получим:

$$
\hat{a}_{\text {out }}(\boldsymbol{\rho}, t)=\sqrt{\lambda_{k}} f(\boldsymbol{\rho}) \psi_{k}(t) \hat{e}_{k}+v a c .
$$

При балансном гомодинном детектировании такого восстановленного сигнала оптимально будет выбрать локальный осциллятор той же пространственно-временной формы:

$$
L(\boldsymbol{\rho}, t)=f(\boldsymbol{\rho}) \psi_{k}(t) .
$$

В силу ортонормированности функций $\left\{\psi_{i}(t)\right\}$ получим фототок следующего вида:

$$
\hat{i}_{0}=\frac{2}{\sqrt{T_{w}}} \sqrt{\lambda_{k}} \hat{x}_{k}
$$

3.2.2. Без учета дифракции, с учетом продольного теплового движения. При наличии продольного теплового движения атомов среды на этапе хранения пространственное распределение записанной в ячейку спиновой когерентности будет им „размыто“. Сигнал на выходе из ячейки памяти окажется суперпозицией собственных мод:

$$
\hat{a}_{\text {out }}(\boldsymbol{\rho}, t)=f(\boldsymbol{\rho}) \sum_{i} \sqrt[4]{\lambda_{i} \lambda_{k}} F_{i k}(\mathbf{q}=0, \overline{\Delta L}) \psi_{i}(t) \hat{e}_{k}+v a c
$$


Примечательно, что операторная часть в правой части при этом сохранится. Несмотря на то, что в силу своей стохастической природы размытие спиновой когерентности из-за теплового движения атомов является необратимым процессом, оно не приводит к потерям возбуждений в атомной среде. Оно приводит лишь к перераспределению этих возбуждений между разными собственными модами атомной среды, которое будет пропорционально квадрату интегралов перекрывания $F_{i k}$.

Оптимальный локальный осциллятор в этом случае будет совпадать по форме с восстановленным импульсом, т. е.

$$
L(\boldsymbol{\rho}, t)=f(\boldsymbol{\rho}) \sum_{j} \sqrt[4]{\lambda_{j} \lambda_{k}} F_{j k}(\mathbf{q}=0, \overline{\Delta L}) \psi_{j}(t)
$$

Отметим, что наличие коэффициента $\sqrt[4]{\lambda_{j} \lambda_{k}} F_{j k}(\mathbf{q}=0, \overline{\Delta L})$ перед каждой функцией $\psi_{j}(t)$ связано с нормировкой корреляционной функции фототока [Mandel], т.е. числом фотоотсчетов, которое зафиксирует фотоприемник за время $T_{w}$ :

$$
N_{\text {count }}=\frac{1}{T_{w}} \int_{0}^{T_{w}} d t \iint_{-\infty}^{+\infty} d \boldsymbol{\rho}|L(\boldsymbol{\rho}, t)|^{2}
$$

Эта нормировка будет минимальна только в том, случае, когда форма локального осциллятора будет полностью совпадать с формой восстановленного поля. Мы учтем эту нормировку при расчетах корреляционных функций в разд. 4.

При таком выборе локального осциллятора детектируемый фототок определяется выражением

$$
\hat{i}_{0}=\frac{2}{\sqrt{T_{w}}} \sum_{i} \sqrt{\lambda_{i} \lambda_{k}} F_{i k}^{2}(\mathbf{q}=0, \overline{\Delta L}) \hat{x}_{k}
$$

3.2.3. Без учета теплового движения, с учетом дифракции. Рассмотрим теперь случай, когда мы учли в модели дифракцию, из-за которой происходит перемешивание исходных квадратурных компонент. Тогда $\hat{x}$-квадратура (и аналогично ей $\hat{y}$-квадратура) поля на выходе ячейки будет включать в себя сжатую $\hat{x}$-квадратуру и растянутую $\hat{y}$-квадратуру поля на входе.

Напомним, что в отсутствие теплового движения атомов при прямом считывании дифракция сводится к набегу постоянной фазы, поэтому в этом случае мы будем интересоваться только обратным считыванием. Запишем выражение для поля на выходе из ячейки памяти:

$$
\hat{a}_{\text {out }}^{\text {back }}(\boldsymbol{\rho}, t)=\sum_{i} \sqrt[4]{\lambda_{i} \lambda_{k}}\left\{F_{i k}^{\text {back }}(\boldsymbol{\rho}) * f(\boldsymbol{\rho})\right\} \psi_{i}(t) \hat{e}_{k}+v a c
$$

Здесь $\{\ldots * \ldots\}$ обозначает свертку функций, зависящих от поперечной координаты $\boldsymbol{\rho}$, а $F_{i k}^{\text {back }}(\boldsymbol{\rho})-$ фурье-образ функции (42). Как и ранее, при детектировании сигнала выберем локальный осциллятор, форма которого совпадает с профилем поля на выходе:

$$
L(\boldsymbol{\rho}, t)=\sum_{j} \sqrt[4]{\lambda_{j} \lambda_{k}}\left\{F_{j k}^{\text {back }}(\boldsymbol{\rho}) * f(\boldsymbol{\rho})\right\} \psi_{j}(t) .
$$

Для такого вида локального осциллятора получим следующее выражение для фототока:

$$
\begin{aligned}
\hat{i}_{0}^{\mathrm{back}}= & \frac{2}{\sqrt{T_{w}}} \sum_{i} \sqrt{\lambda_{i} \lambda_{k}} \\
& \times\left(\iint_{-\infty}^{+\infty} d \boldsymbol{\rho}\left|\left\{F_{i k}^{\mathrm{back}}(\boldsymbol{\rho}) * f(\boldsymbol{\rho})\right\}\right|^{2}\right) \hat{x}_{k} .
\end{aligned}
$$

Отметим, что выбор правильной формы локального осциллятора при перемешивании квадратур из-за наличия дифракции носит исключительно важное значение.

Для сравнения рассмотрим, что получится, если мы пренебрежем дифракцией, и при детектировании возьмем рассмотренный ранее локальный осциллятор (51). В результате в фототоке появится вклад не только от сжатой квадратуры, но и от растянутой:

$$
\begin{aligned}
\hat{i}_{0}^{\text {back }}= & \frac{2}{\sqrt{T_{w}}} \lambda_{k}\left(\iiint_{-\infty}^{+\infty} d \boldsymbol{\rho} \operatorname{Re}\left[\left\{F_{k k}^{\mathrm{back}}(\boldsymbol{\rho}) * f(\boldsymbol{\rho})\right\}\right]\right) \hat{x}_{k} \\
& \left.+\left(\iint_{-\infty}^{+\infty} d \boldsymbol{\rho} \operatorname{Im}\left[\left\{F_{k k}^{\mathrm{back}}(\boldsymbol{\rho}) * f(\boldsymbol{\rho})\right\}\right]\right) \hat{y}_{k}\right) .
\end{aligned}
$$

В этом выражении мы обозначили с помощью $\operatorname{Re}[. .$.$] и$ $\operatorname{Im}[. .$.$] реальную и мнимую части выражений, стоящих$ в скобках. Таким образом, детектирование сигнала с таким локальным осциллятором приведет к ухудшение квантово-статистических свойств восстановленного сигнала, вызванного наличием растянутой квадратуры $\hat{y}$, а то и вовсе к их полной потере. То же самое можно сказать и про выбор любой другой формы локального осциллятора, кроме (59), и чем больше степень сжатия $\hat{x}$-квадратуры и растяжения $\hat{y}$-квадратуры, тем большее влияние оказывает выбор правильной формы локального осциллятора при гомодинировании.

\subsection{4. Общие правила при выборе локального} осциллятора. Из рассмотренных выше примеров, а также результатов наших расчетов, которые мы приведем в следующей части статьи, можно заключить, что в одномодовом режиме оптимальной формой локального осциллятора при детектировании является форма восстановленного из ячейки памяти сигнала. Полученный вывод является ожидаемым. В процессе памяти при распространении сигнального поля внутри атомной среды при наличии дифракции и теплового движения сигнальное поле не только меняет свой временной профиль, но и приобретает неоднородный, зависящий от времени фазовый набег. И чтобы подавить возникший фазовый набег, согласно выражению (48), проще всего 
взять локальный осциллятор, полностью совпадающий с восстановленным сигналом. Вместе с тем наличием дифракции в теоретических расчетах очень часто пренебрегают. Действительно, в случае, когда выполняется условие $\mathbf{q}^{2} L / k_{s} \ll 1$, выражение перед $\hat{y}$-квадратурой в (60) будет мало. Однако из-за того, что $\hat{y}$-квадратура является растянутой, ее вклад при детектировании может оказаться существенным.

Мы не проанализировали здесь ситуацию, когда учитываются и дифракция, и тепловое движение, но все выводы, сделанные ранее, будут справедливы в том числе и для такой конфигурации. Влияние поперечного движения рассмотрим при анализе численных расчетов.

\section{3. Гомодинное детектирование сигнала от субпуассоновского лазера}

Рассмотрим теперь, как происходит гомодинное детектирование в случае, когда в ячейку памяти был записан сигнал от субпуассоновского лазера с захватом фазы. Теперь нас будет интересовать сжатие во всем диапазоне частот, а не только на нулевой частоте, поэтому будем следить за спектром сжатия флуктуаций фототока

$$
\left\langle\delta \hat{i}_{\omega} \delta \hat{i}_{-\omega}\right\rangle=\frac{1}{T_{w}} \int_{0}^{T_{w}} \int_{0}^{T_{w}} d t d t^{\prime} e^{-i \omega\left(t-t^{\prime}\right)}\left\langle\delta \hat{i}(t) \delta \hat{i}\left(t^{\prime}\right)\right\rangle .
$$

Сами фототоки и вся процедура гомодинирования будут по-прежнему определяться выражением (48).

Учтем, что в отличие от рассмотренного ранее модельного одномодового источника субпуассоновский лазер в базисе собственных мод $\left\{\psi_{i}(t)\right\}$ является многомодовым источником. Как мы увидим, главной особенностью многомодового источника будет то, что развитие каждой его моды в процессе памяти при наличии дифракции приведет к появлению у каждой из них собственных фазовых набегов. Это сделает невозможным подбор формы локального осциллятора простым повторением формы поля на выходе из ячейки, как для одномодового режима.

Рассмотрим явный вид получающихся выражений для этой и других конфигураций памяти в случае многомодового источника.

\subsection{1. Без учета дифракции и теплового движе-}

ния. В случае, когда нет ни дифракции, ни теплового движения, квадратуры не перемешиваются, а поле на выходе ячейки памяти имеет вид

$$
\hat{a}_{\text {out }}(\boldsymbol{\rho}, t)=f(\boldsymbol{\rho}) \sum_{i} \sqrt{\lambda_{i}} \psi_{i}(t) \hat{e}_{i}+v a c,
$$

оптимальным выбором формы локального осциллятора будет по-прежнему та, что совпадает с формой поля на выходе из ячейки:

$$
L(\boldsymbol{\rho}, t)=f(\boldsymbol{\rho}) \sum_{j} \sqrt{\lambda_{j}} \psi_{j}(t)
$$

Флуктуации фототока при детектировании такого сигнала будут описываться выражением

$$
\delta \hat{i}(t)=2 \sum_{i j} \sqrt{\lambda_{i} \lambda_{j}} \psi_{i}(t) \psi_{j}(t) \delta \hat{x}_{i}
$$

\subsection{2. Без учета дифракции, с учетом продоль-} ного теплового движения. Для случая, когда мы учитываем только продольное тепловое движение атомов на этапе хранения сигнальное поле после прохождения ячейки памяти, согласно (34), будет описываться выражением

$$
\hat{a}_{\text {out }}(\boldsymbol{\rho}, t)=f(\boldsymbol{\rho}) \sum_{i j} \sqrt[4]{\lambda_{i} \lambda_{j}} F_{i j}(\mathbf{q}=0, \overline{\Delta L}) \psi_{i}(t) \hat{e}_{j}+v a c
$$

Для такого поля оптимальная форма локального осциллятора примет вид

$$
L(\boldsymbol{\rho}, t)=f(\boldsymbol{\rho}) \sum_{k l} \sqrt[4]{\lambda_{k} \lambda_{l}} F_{k l}(\mathbf{q}=0, \overline{\Delta L}) \psi_{k}(t)
$$

а флуктуации фототока при этом будут равны

$$
\delta \hat{i}(t)=2 \sum_{i j k l} \sqrt[4]{\lambda_{i} \lambda_{j}} F_{i j}(\mathbf{q}=0, \overline{\Delta L}) \sqrt[4]{\lambda_{k} \lambda_{l}} F_{k l} \psi_{i}(t) \psi_{k}(t) \delta \hat{x}_{j}
$$

Несмотря на то, что выражение для фототока включает в себя целых четыре суммирования, это выражение в целом аналогично тому, что мы получили для одномодового случая в той же конфигурации памяти.

\subsection{3. С учетом дифракции, без учета теплово-} го движения. Рассмотрим теперь ситуацию, когда в модель включена дифракция. При обратном считывании сигнал на выходе ячейки будет иметь следующий вид:

$$
\hat{a}_{\text {out }}^{\text {back }}(\boldsymbol{\rho}, t)=\sum_{i j} \sqrt[4]{\lambda_{i} \lambda_{j}}\left\{F_{i j}^{\text {back }}(\boldsymbol{\rho}) * f(\boldsymbol{\rho})\right\} \psi_{i}(t) \hat{e}_{j}+v a c
$$

В данном выражении сумма по индексу $i$ перед оператором $\hat{a}_{j}$ определяет не только временной профиль, но также и фазовый набег $j$-й моды при ее восстановлении из ячейки памяти. Причем, так как функции перекрывания $F_{i j}^{\mathrm{back}}(\boldsymbol{\rho})$ не равны друг другу, для каждой восстановленной моды этот набег будет свой.

Если в качестве локального осциллятора выбрать сигнал по форме, совпадающий с полем на выходе:

$$
L(\boldsymbol{\rho}, t)=\sum_{k l} \sqrt[4]{\lambda_{k} \lambda_{l}}\left\{F_{k l}^{\mathrm{back}}(\boldsymbol{\rho}) * f(\boldsymbol{\rho})\right\} \psi_{k}(t)
$$


то после гомодинирования сигнала с таким локальным осциллятором флуктуации фототока будут равны:

$$
\begin{aligned}
\delta \hat{i}^{\mathrm{back}}(t)= & 2 \sum_{i j k l} \sqrt[4]{\lambda_{i} \lambda_{j}} \sqrt[4]{\lambda_{k} \lambda_{l}} \psi_{i}(t) \psi_{k}(t) \\
& \times\left(\iiint_{-\infty}^{+\infty} d \boldsymbol{\rho} \operatorname{Re}\left[\mathscr{F}_{i j k l}^{\mathrm{back}}(\boldsymbol{\rho})\right]\right) \delta \hat{x}_{j} \\
& \left.+\left(\iint_{-\infty}^{+\infty} d \boldsymbol{\rho} \operatorname{Im}\left[\mathscr{F}_{i j k l}^{\text {back }}(\boldsymbol{\rho})\right]\right) \delta \hat{y}_{j}\right),
\end{aligned}
$$

где введено обозначение

$\mathscr{F}_{i j k l}^{\text {back }}(\boldsymbol{\rho})=\left\{F_{i j}^{\text {back }}(\boldsymbol{\rho}) * f(\boldsymbol{\rho})\right\}\left\{\left(F_{k l}^{\text {back }}(\boldsymbol{\rho})\right)^{*} * f(\boldsymbol{\rho})\right\}$.

В найденном выражении для флуктуаций фототока коэффициент перед флуктуациями $\hat{y}$-квадратуры обращается в нуль только при $i=k, j=l$ или $i=l, j=k$. В связи с этим в спектре будут присутствовать избыточные шумы, связанные с подмешиванием растянутой квадратуры. При прямом считывании такой проблемы, естественно, не возникает.

Как мы показали, в многомодовом случае при обратном считывании дифракция не позволяет взять локальный осциллятор с формой, повторяющей поле на выходе из ячейки. Однако даже для такого источника существуют методы оптимизации протокола, которые позволят детектировать сигнал после ячейки памяти без добавления избыточного шума. О них мы скажем в заключении к этой статье.

Отметим, что, как и для одномодового случая, мы не привели здесь явных выражений для конфигураций, в которых учитываются и продольное движение, и дифракция, их анализ в целом повторяет выводы, сделанные нами ранее. Также стоит сказать, что поперечное движение не приводит к перемешиваю собственных мод среды, как это делает продольное, однако это не значит, что поперечное движение не будет оказывать никакого влияния на процесс детектирования, что мы увидим при обсуждении результатов, полученных из численных расчетов.

\section{4. Численный анализ для одномодового и многомодового излучения}

Чтобы проверить наши выводы о роли теплового движения и дифракции при сохранении в ячейке памяти квадратурно-сжатого света, мы провели численный эксперимент, в котором в качестве параметров взяли следующие безразмерные величины: $T_{w}=10, L=12$, $w_{0}=0.072$ и $k_{s}=2 \pi / \lambda=9 \cdot 10^{3}$. В размерных величинах это соответствует длине ансамбля $L=1 \mathrm{~cm}$, оптической толщине $2 g^{2} N L / \gamma=120$, ширине перетяжки гауссова пучка $w_{0}=60 \mu$ и длине волны света $\lambda=580 \mathrm{~nm}$. Сжатие сигнального поля на входе в ячейку для одномодового и многомодового источников взяли равным $-10 \mathrm{~dB}$. Для удобства ввели дифракционный параметр $q_{0}=1 / w_{0}$, при этом случаю, когда дифракции нет, отвечает $q_{0}=0$ (плоская волна).

Для безразмерных оптической толщине $L=12$ и времени записи $T_{w}=10$ для прямого и для обратного считывания получаются следующие собственные числа: $\lambda_{1}=0.89, \lambda_{2}=0.51$ и $\lambda_{3}=0.16$ - для прямого считывания; $\lambda_{1}=1.00, \lambda_{2}=0.99$ и $\lambda_{3}=0.67-$ для обратного. Остальные собственные числа пренебрежимо малы, поэтому мы можем считать, что в процессе памяти участвуют только первые три собственные функции, т.е. ряд (25) может быть ограничен тремя первыми членами.

\section{1. Одномодовый источник сжатого излучения}

Рассмотрим сначала результаты, которые мы получили для одномодового модельного источника. Они представлены в виде гистограмм на рис. 3. В верхнем ряду этого рисунка собраны результаты для случая обратного считывания, а в нижнем - для прямого. При этом первые гистограммы в нижнем и верхнем ряду относятся к первым собственным функция, вторые ко вторым, а третьи - к третьим. В каждой из этих гистограмм первый столбец (a) отвечает величине сжатия сигнального поля на входе в ячейку памяти $(-10 \mathrm{~dB})$, a все остальные $(\mathrm{b}-1)$ разным конфигурациям памяти, в которых мы учитывали влияние теплового движения атомов и дифракции. При этом в столбцах (b) для сравнения приведены результаты для конфигурации, когда и дифракция, и тепловое движение атомов отсутствуют. Фактически высота этого столбца определяется лишь квадратом собственного числа [26], отвечающего данной собственной функции, и степенью сжатия поля на входе в ячейку. Таким образом, видно, что квадрат собственного значения или эффективность памяти является своего рода верхней границей для одномодового случая: все остальные столбцы (c-l) будут ниже столбца (b). Мы видим, что в целом при обратном считывании эффективность памяти при отсутствии движения и дифракции оказывается выше, как этого и следовало ожидать [18].

Далее следуют столбцы (c) и (d), которые показывают величину сжатия при наличии продольного теплового движения атомов, когда среднее смещение атомов на этапе хранения составляет 1/6 длины и полную длину ячейки. Как и в работе [15], мы получили, что продольное тепловое движение атомов тем больше влияет на процес хранения, чем более сложным оказывается профиль собственной функции и отвечающей ей моды атомной среды. Так, наиболее устойчивой модой к продольному тепловому движению при обратном считывании оказалась вторая мода, а при прямом - первая. Этим модам соответствуют гладкие пространственные профили.

За ними следуют столбцы (e) и (f). Эти столбцы показывают, как изменится сжатие, если включить в модель дифракцию, причем столбец (е) соотвествует 

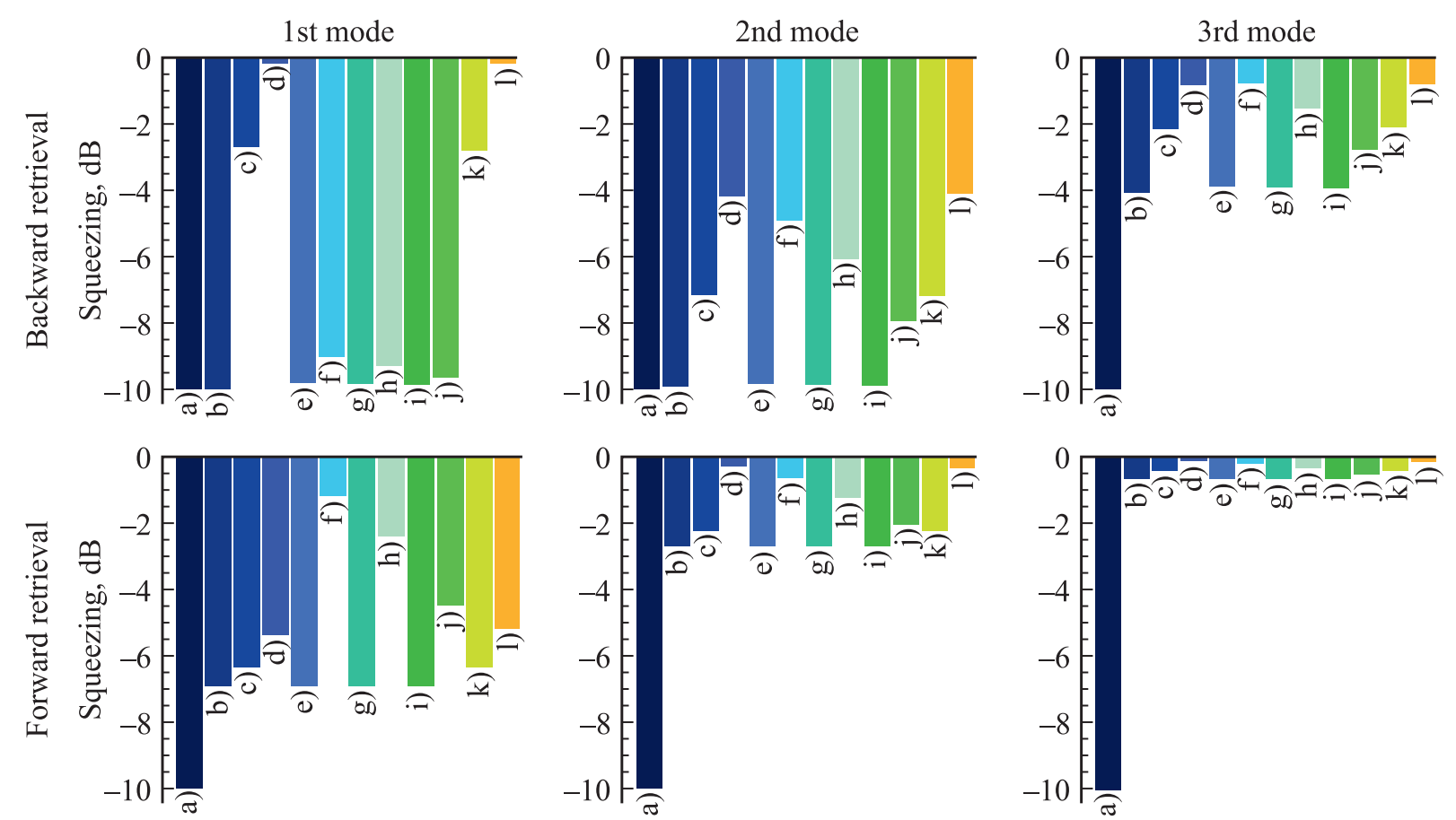
a) input;
e) $\left(\overline{\Delta L}=0, \overline{\Delta w}=0, q_{0}=40\right)$, "good" LO;
i) $\left(\overline{\Delta L}=0, \overline{\Delta w}=0.07, q_{0}=40\right)$, "good" LO;
b) $\left(\overline{\Delta L}=0, \overline{\Delta w}=0, q_{0}=0\right)$;
f) $\left(\overline{\Delta L}=0, \overline{\Delta \bar{w}}=0, q_{0}=40\right)$, "bad" LO;
j) $\left(\overline{\Delta L}=0, \overline{\Delta w}=0.07, q_{0}=40\right)$, "bad" LO;
c) $\left(\overline{\Delta L}=2, \overline{\Delta w}=0, q_{0}=0\right)$
g) $\left(\overline{\Delta L}=0, \overline{\Delta w}=0.03, q_{0}=40\right)$, "good" LO;
k) $\left(\overline{\Delta L}=2, \overline{\Delta w}=0, q_{0}=40\right)$, "good" LO;
d) $\left(\overline{\Delta L}=12, \overline{\Delta w}=0, q_{0}=0\right)$;
h) $\left(\overline{\Delta L}=0, \overline{\Delta w}=0.03, q_{0}=40\right)$, "bad" LO;
1) $\left(\overline{\Delta L}=12, \overline{\Delta w}=0, q_{0}=40\right)$, "bad" LO.

Рис. 3. Степень сжатия сигнального поля с профилем одной из собственных функций на входе в ячейку памяти (а) и на выходе из нее $(\mathrm{b}-1)$ при различных параметрах: $\overline{\Delta L}-$ среднее продольное смещение, $\overline{\Delta w}$ - среднее поперечное смещение, $q_{0}-$ дифракционный параметр. „bad LO“ - при неоптимальном выборе локального осциллятора; „good LO“ — при оптимальном.

случаю, когда выбран оптимальный локальный осциллятор, учитывающий дифракцию, а столбец (f), когда форма локального осциллятора при гомодинировании совпадает с сигналом, который был подан на вход ячейки памяти, т.е. неоптимальна. Как мы видим, при наших параметрах сжатие остается в каждом случае, но при неоптимальном выборе локального осциллятора потери из-за подмешивания растянутой квадратуры могут быть огромными.

Столбцы $(\mathrm{g}-\mathrm{j})$ отвечают случаям, когда есть и дифракция, и поперечное тепловое движение атомов среды на этапе хранения. Как мы уже отмечали, наличие поперечного теплового движения в случае одномодового гауссова пучка приводит к появлению в (38) нового поперечного профиля, представляющего собой ту же гауссову функцию, но с большей полушириной. Фактически это означает, что при считывании наш сигнал будет размазан по большей площади, но при этом его квантово-статистические свойства никак не изменятся, так как нет потерь. Однако из диаграмм видно, что высота столбцов неодинакова. Чтобы понять причину этого, давайте на каждой гистограмме рассмотрим столбцы (h) и (j). В отличие от (g) и (i) они отвечают тем случаям, когда локальный осциллятор при гомодинировании выбран неоптимальным образом и дифракция не подавлена.
Мы видим, что высота столбца (j), который соответствует большему среднему поперечному смещению атомов, выше, чем (h), т. е. сжатие для него больше. Это говорит нам о том, что чем более плоским является поперечный профиль сигнального поля, и чем ближе он к плоской волне, тем меньшее влияние будеть иметь дифракция. В этом можно убедиться, если вместе со столбцами (h) и (j) рассматреть еще столбец (f), когда дифракция также не подавлена выбором локального осциллятора, но движение при этом отсутствует.

Наконец, последних два столбца (k) и (1) показывают, что происходит с сжатием при продольном движении и дифракции. При этом, как и раньше, эти случаи отличаются выбором локального осциллятора при гомодинировании. Для такой конфигурации перемешивание между собственными функциями является наиболее сильным, и как следствие самым деструктивным, так как перемешивание мод атомной среды приведет к тому, что сигнал будет перераспределен между большим количеством собственных мод, часть из которых имеет низкую эффективность при считывании. Выбор локального осциллятора, подавляющего дифракцию при гомодинировании, позволяет частично уменьшить эти потери. 
В целом из анализа представленных диаграмм можно сделать вывод, что продольное тепловое движение является наиболее деструктивным фактором при сохранении сжатого света в ячейке памяти. При неправильном выборе локального осциллятора из-за дифракции могут возникнуть большие потери. Кроме того, все результаты будут во многом зависеть от формы временного профиля сигнального поля и эффективности его записи и считывания (собственного числа). Также примечательно, что для случая обратного считывания полученные результаты в целом лучше, чем для прямого.

\section{2. Многомодовый источник сжатого излучения}

Рассмотрим теперь результаты, которые получились у нас при численном расчете для многомодового источника излучения. Напомним, что в качестве такого источника мы выбрали субпуассоновский лазер с захватом фазы.

На рис. 4 представлены спектры сжатия флуктуаций фототока для случая обратного считывания (левый рисунок) и случая прямого считывания (правый рисунок). Пунктирной черной кривой, расположенной ниже всех остальных, показан спектр сжатия излучения, поданного на вход ячейки памяти. Мы видим, что по сравнению с этой кривой все остальные, которые отвечают различным режимам хранения в ячейке, оказываются значительно выше. Это связано с тем, что в разложении прямоугольного профиля импульса субпуассоновского лазера участвуют в том числе и такие функции $\psi_{i}(t)$, которым отвечают пренебрежимо малые собственные числа $\lambda_{i}$. Тем не менее на нулевой частоте сжатие достаточно большое, так как наибольший вес в этом разложении дают первые две собственные функции, которым отвечают наиболее гладкие моды среды и самые большие собственные числа.

Для всех рассмотренных случаев мы выбирали локальный осциллятор при гомодинировании оптимальным, именно поэтому на нулевой частоте влияние дифракции почти никак не проявляется. Однако, дифракция в спектре будет проявляться на „крыльях“, полученных распределений, т.е. для частот, отличных от нулевой. Это является следствием того, что в выражении (48) для спектров фототока стоит еще одна комплексная экспонента (от преобразования Фурье), которая несколько ухудшает подобранный нами локальный осциллятор для ненулевых частот. Таким образом, для отдельных частотных компонент сжатие несколько ухудшится, но сжатие сигнала как целого (при $\omega=0$ ) останется неизменным. Как следствие, ширина информационного канала, в который входит рассматриваемая нами ячейка памяти, при наличии дифракции и продольного теплового движения незначительно уменьшится.

В целом, как мы видно из графиков, выводы, которые мы сделали для одномодового случая, повторяются и для многомодового случая. Наиболее деструктивным фактором и при этом выступает продольное тепловое движение атомов среды на этапе хранения. Однако наличие дифракции и правильный выбор гомодина играют далеко не последнюю роль.

\section{5. Пути оптимизации процесса хранения при наличии дифракции и теплового движения}

Одним из возможных вариантов оптимизации хранения квадратурно-сжатого света является учет негативных эффектов при нахождении собственных функций памяти, т.е. замена полного набора (базиса) функций $\left\{\psi_{i}(t)\right\}$, с которым мы работали раньше, более подходящим. Получим и рассмотрим два таких базиса.

\section{1. Моды памяти с учетом теплового движения}

Из анализа полученных результатов мы знаем, что наиболее деструктивным фактором при сохранении квадратурно-сжатого света является продольное тепловое движение атомов среды. В связи с этим мы можем включить тепловое движение в ядро для полного цикла памяти при отсутствии дифракции и поставить для него задачу на собственные функции и значения. Такая техника оптимизации была предложена в работе [15]

$$
\sqrt{\lambda_{i}} \psi_{i}^{\overline{\Delta L}}(t)=\int_{0}^{T_{w}} d t^{\prime} G\left(t, t^{\prime} ; \overline{\Delta L}\right) \psi_{i}^{\overline{\Delta L}}\left(t^{\prime}\right) .
$$

Ядро $G\left(t, t^{\prime} ; \overline{\Delta L}\right)$ по-прежнему является симметричным относительно перестановки своих временных аргументов, и поэтому такая постановка задачи оказывается возможной.

На рис. 5, $а$ приведены полученные результаты. Отметим, что при включении теплового движения в ядро можно подобрать профиль сигнального поля таким образом (первые диаграммы в верхнем и в нижнем ряду), что система оказывается более устойчива к тепловому движению (для заданного $\overline{\Delta L}$ ). Это видно при сравнении двух последних столбцов (k) и (1) с этими же столбцами на рис. 3. Наличие дифракции при этом не оказывает сильного влияния на сохранение сжатия, потому что правильный подбор локального осциллятора позволяет полностью подавить вклад от растянутой квадратуры.

\section{2. Моды памяти с учетом дифракции}

Другая возможность улучшить сохранение сжатия найти собственные функции памяти с учетом влияния дифракции. Эта задача актуальна только для случая обратного считывания, при котором дифракционный множитель находится внутри ядра памяти. Для каждого фиксированного q ядро памяти $G^{\mathrm{back}}\left(\mathbf{q}, t, t^{\prime}\right)$ симметрично по перестановке аргументов $t$ и $t^{\prime}$, поэтому для него 

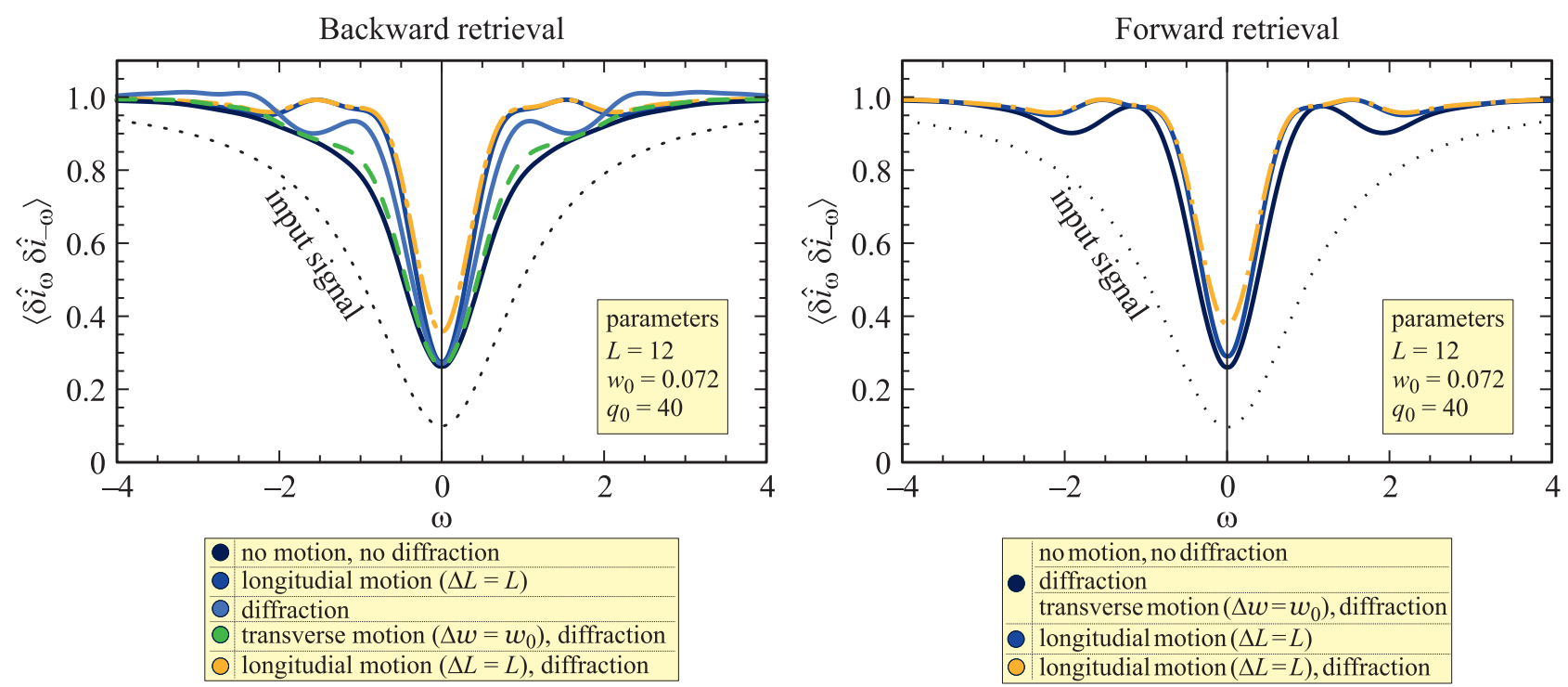

Рис. 4. Спектр сжатия сигнального поля от субпуассоновского лазера при различных параметрах.
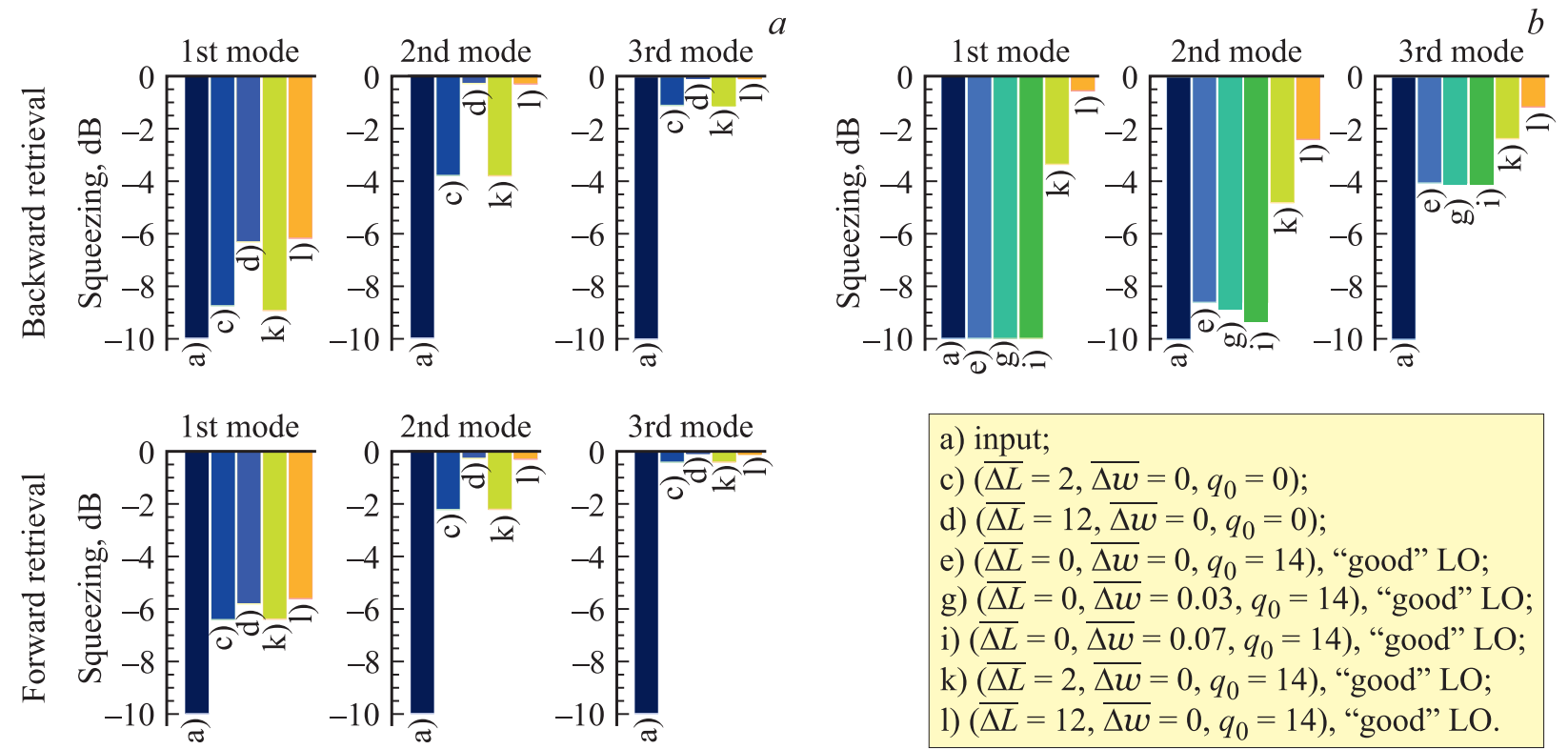
a) input;
c) $\left(\overline{\Delta L}=2, \overline{\Delta w}=0, q_{0}=0\right)$
d) $\left(\overline{\Delta L}=12, \overline{\Delta w}=0, q_{0}=0\right)$;
e) $\left(\overline{\Delta L}=0, \overline{\Delta w}=0, q_{0}=14\right)$, "good" LO;
g) $\left(\overline{\Delta L}=0, \overline{\Delta w}=0.03, q_{0}=14\right)$, "good" LO;
i) $\left(\overline{\Delta L}=0, \overline{\Delta w}=0.07, q_{0}=14\right)$, "good" LO;
k) $\left(\overline{\Delta L}=2, \overline{\Delta w}=0, q_{0}=14\right)$, "good" LO;
1) $\left(\overline{\Delta L}=12, \overline{\Delta w}=0, q_{0}=14\right)$, “good" LO.

Рис. 5. Степень сжатия сигнального поля с профилем одной из собственных функций, найденных с учетом: $a$ ) продольного теплового движения; $b$ ) с учетом дифракции.

также может быть поставлена задача на собственные функции и собственные значения:

$$
\sqrt{\lambda_{i}(\mathbf{q})} \psi_{i}^{\mathrm{dif}}(\mathbf{q}, t)=\int_{0}^{T_{w}} d t^{\prime} G^{\mathrm{back}}\left(\mathbf{q}, t, t^{\prime}\right) \psi_{i}^{\mathrm{dif}}\left(\mathbf{q}, t^{\prime}\right) .
$$

Как видно из рис. 5, $b$, на котором приведены полученные результаты, можно полностью подавить дифракцию для первой моды, а также для двух других. Однако, найденные моды оказываются сильно подвержены влиянию продольного теплового движения, и даже при относительно небольших средних продольных смещениях ато- мов сжатие всех мод значительно падает (стоблцы $(\mathrm{k})$ и (1)).

\section{6. Заключение}

В работе проанализировано влияние дифракции и теплового движения атомов среды на этапе хранения для протокола быстрой резонансной многомодовой квантовой памяти. При этом в качестве источников квадратурно-сжатого излучения рассмотрены модельный одномодовый источник и субпуассоновский лазер с захватом фазы, который в базисе собственных функций памяти 
при наших параметрах уже является многомодовым источником.

Показано, что и дифракция, и продольное тепловое движение играют значительную роль при сохранении квадратурно-сжатого света. В частности, их наличие приводит к перемешиванию собственных мод ячейки, т. е. перераспределению между ними образовавшихся на этапе записи спиновых возбуждений. Кроме того, наличие дифракции также приводит к перемешиванию квадратур, и $\hat{x}$-квадратура поля на выходе будет суммой сжатой $\hat{x}$-квадратуры и растянутой $\hat{y}$-квадратуры поля на входе в ячейку памяти, взятых с некоторыми определенными коэффициентами.

Эти два фактора существенно ухудшают квантовостатистические свойства восстановленного излучения, если не брать их во внимание. Однако выбор правильной формы локального осциллятора при процедуре гомодинного детектирования позволяет частично или полностью подавить перемешивание квадратур, поэтому наиболее деструктивным оказывается перемешивание собственных мод, так как при этом происходит перераспределение в том числе и по тем модам, которым отвечает невысокая эффективность считывания.

Поперечное движение, напротив, не сказывается на процессе памяти при правильном подборе формы локального осциллятора при гомодинировании. Впрочем, в нашей задаче поперечная координата не являлась квантовой степенью свободы, т.е. в поперечной плоскости поле на входе ячейки не содержало в себе каких-либо квантовых корреляций.

Также мы рассмотрели два возможных способа оптимизации протокола, включив в ядро полного цикла памяти сначала продольное тепловое движение, а затем и дифракцию. Для каждого из этих случаев мы получили собственный базис и рассмотрели, как они будут вести себя при наличии и дифракции, и продольного теплового движения. Мы увидели, что базис, учитывающий дифракцию, оказывается не устойчив к тепловому движению. Напротив, базис, в котором было учтено продольное тепловое движение, оказался более устойчив к дифракции, так как последнюю можно, как и в ранее рассмотренных случаях, подавить с помощью правильной формы локального осциллятора при гомодинировании.

Для многомодового источника полностью подавить дифракцию путем выбора формы локального осциллятора оказалось невозможно. Однако в нашей работе мы рассмотрели наиболее простую форму управляющего поля, когда оно является плоской монохроматичной волной. В работах [27-30] было показано, как можно подобрать форму управляющего поля, чтобы записать сигнал с произвольным заданным временным профилем. На языке собственных функций это означает, что при правильно подобранном управляюшем поле одна из собственных функций будет совпадать с временным профилем сигнала на входе в ячейку, т. е. задача сведется к одномодовой, для которой дифракцию можно подавить при гомодинировании.

\section{Финансирование работы}

Работа выполнена при финансовой поддержке Российского фонда фундаментальных исследований (грант № 18-02-00648 и грант № 19-02-00204).

\section{Конфликт интересов}

Авторы заявляют, что у них нет конфликта интересов.

\section{Список литературы}

[1] Hammerer K., Sørensen A.S., Polzik E.S. // Rev. Mod. Phys. 2010. V. 82. P.1041. doi 10.1103/Rev. Mod. Phys. 82.1041

[2] Lvovsky A.I., Sanders B.C., Tittel W. // Nat. Photon. 2009. V. 3. P. 706. doi 10.1038/nphoton.2009.231

[3] Simon J., Tanji H., Thompson J.K., Vuleti'c V. // Phys. Rev. Lett. 2007. V. 98. P. 183601. doi 10.1103/PhysRevLett.98.183601.

[4] Chou C.-W., Laurat L., Deng H., Choi K.S., de Riedmatten H., Felinto D., Kimble H.J. // Science 2007. P. 316. V. 1316. doi 10.1126/science. 1140300

[5] Chen Y.-A., Chen S., Yuan Z.S., Zhao B., Chuu C.S., Schmiedmayer J., Pan J.-W. // Nat. Phys. 2008. V. 4. P. 103. doi $10.1038 /$ nphys 832

[6] Tittel W., Afzelius M., Chaneliere T., Cone R.L., Kroll S., Moiseev S.A., Sellars M. // Laser Photon. Rev. 2010. V. 4. P. 244. doi 10.1002/lpor.200810056

[7] Hosseini M., Campbell G., Sparkes B.M., Lam P.K., Buchler B.C. // Nat. Phys. 2011. V. 7. P. 794. doi 10.1038/nphys2021

[8] Camacho R.M., Vudyasetu P.K., Howell J.C. // Nat. Photon. 2009. V. 3. P. 103. doi doi.org/10.1038/nphoton.2008.290

[9] Phillips N.B., Gorshkov A.V., Novikova I. // Phys. Rev. A 2011. V. 83. P. 063823. doi 10.1103/PhysRevA.83.063823

[10] de Almeida A.J.F., Sales J., Maynard M.-A., Lauprêtre T., Bretenaker F., Felinto D., Goldfarb F., Tabosa J.W.R. // Phys. Rev. A 2014. V. 90. P. 043803. doi 10.1103/PhysRevA.90.043803

[11] Brannan T., Qin Z., Mac Rae A., Lvovsky A.I. // Opt. Lett. 2014. V. 39. P. 18 doi $10.1364 /$ OL.39.005447

[12] Novikova I., Walsworth R.L., Xiao Y. // Laser Photon. Rev. 2011. V. 6. P. 333. doi 10.1002/lpor.201100021

[13] Borregaard J., Zugenmaier M., Petersen J.M., Shen H., Vasilakis G., Jensen K., Polzik E. S., Sørensen A.S. // Nat. Comm. 2016. V. 7. P. 11356. doi $10.1038 /$ ncomms 11356

[14] Surmacz K., Nunn J., Reim K., Lee K.C., Lorenz V.O., Sussman B., Walmsley I.A., Jaksch D. // Phys. Rev. A 2008. V. 78. P. 033806 doi 10.1103/PhysRevA.78.033806

[15] Tikhonov K., Golubeva T., Golubev Y. // Eur. Phys. J. D 2015. V. 69. P. 252. doi 10.1140/epjd/e2015-60370-6 
[16] Vernaz-Gris P., Tranter A.D., Everett J.L., Leung A.C., Paul K.V., Campbell G.T., Lam P.K., Buchler B.C. // Opt. Expr. 2018. V. 26. N 10. P. 12424. doi 10.1364/OE.26.012424

[17] Golubeva T.Y., Golubev Y.M., Mishina O., Bramati A., Laurat J., Giacobino E. // Phys. Rev. A 2011. V. 83. P. 053810. doi 10.1103/PhysRevA.83.053810

[18] Golubeva T.Y., Golubev Y.M., Mishina O., Bramati A., Laurat J., Giacobino E. // Eur. Phys. J. D 2012. V. 66. P. 275. doi 10.1140/epjd/e2012-20723-3

[19] Karg T.M., Gouraud B., Ngai C.T., Schmid G.-L., Hammerer K., Treutlein P. Light-mediated strong coupling between a mechanical oscillator and atomic spins 1 meter apart // Science 2020 [Электронный ресурс] Режим доступа: https://doi.org/10.1126/science.abb0328

[20] Gorshkov A.V., Andre A., Lukin M.D., Sorensen A.S. // Phys. Rev. A 2007. V. 76. P. 033804. doi 10.1103/PhysRevA.76.033804

[21] Metcalf H.F. Laser Cooling and Trapping. New York: Springer-Verlag, 1999. $323 \mathrm{p}$.

[22] Kolobov M.I. // Rev. Mod. Phys. 1999. V. 71. N 5. P. 1539. doi 10.1103/RevModPhys.71.1539

[23] Fedorov M.V. // Phys. Scr. 2020. V. 95. P. 6. doi 10.1088/1402-4896/ab7aa7

[24] Golubeva T.Y., Ivanov D.A., Golubev Y.M. // Phys. Rev. A 2008. V. 77. P. 052316. doi 10.1103/PhysRevA.77.052316

[25] Mandel L., Wolf E. Optical Coherence and Quantum Optics. Cambridge University Press, 2014. 1194 р.; Мандель Л., Воль $\varnothing$ Э. Оптическая когерентность и квантовая оптика. М.: Физматлит, 2000. 896 с.

[26] Golubeva T.Y., Golubev Yu.M. // J. Russ. Laser Res. 2015. V. 36. P. 522. doi 10.1007/s10946-015-9531-y

[27] Novikova I., Gorshkov A.V., Phillips D.F., Sфrensen A.S., Lukin M.D., Walsworth R.L. // Phys. Rev. Lett. 2007. V. 98. P. 243602. doi doi.org/10.1103/PhysRevLett.98.243602

[28] Novikova I., Phillips N.B., Gorshkov A.V. // Phys. Rev. A 2007. V. 78. P. 021802(R). doi 10.1103/PhysRevA.78.021802

[29] Кузьмин В.В., Ветлугин А.Н., Соколов И.В. // Опт. и спектр. 2015. Т. 119. № 6. С. 1000; Kuzmin V.V., Vetlugin A.N., Sokolov I.V. // Opt. Spectrosc. 2015. V. 119, P. 1004. doi 10.1134/S0030400X15120152

[30] Manukhova A.D., Tikhonov K.S., Golubeva T.Yu., Golubev Yu.M. // Phys. Rev. A 2017. V. 96. P. 023851. doi 10.1103/PhysRevA.96.023851 